\title{
Design and characterization of the Large-aperture Experiment to Detect the Dark Age (LEDA) radiometer systems
}

\author{
D. C. Price, ${ }^{1,2,3 \star}$ L. J. Greenhill, ${ }^{1}$ A. Fialkov, ${ }^{1}$ G. Bernardi, ${ }^{4,5}$ H. Garsden, ${ }^{1}$ \\ B. R. Barsdell, ${ }^{6}$ J. Kocz, ${ }^{1,7}$ M. M. Anderson, ${ }^{7}$ S. A. Bourke, ${ }^{7}$ J. Craig, ${ }^{8}$ M. R. Dexter, ${ }^{2}$ \\ J. Dowell, ${ }^{8}$ M. W. Eastwood, ${ }^{7}$ T. Eftekhari, ${ }^{1,8}$ S. W. Ellingson, ${ }^{9}$ G. Hallinan, ${ }^{7}$ \\ J. M. Hartman, ${ }^{7}$ R. Kimberk, ${ }^{1}$ T. Joseph W. Lazio, ${ }^{10}$ S. Leiker, ${ }^{1}$ D. MacMahon, ${ }^{2}$ \\ R. Monroe, ${ }^{7}$ F. Schinzel, ${ }^{8,11}$ G. B. Taylor, ${ }^{8}$ E. Tong, ${ }^{1}$ D. Werthimer ${ }^{2}$ and D. P. Woody ${ }^{7}$ \\ ${ }^{1}$ Harvard-Smithsonian Center for Astrophysics, 60 Garden Street, Cambridge, MA 02138, USA \\ ${ }^{2}$ University of California Berkeley, 501 Campbell Hall, Berkeley, CA 94720, USA \\ ${ }^{3}$ Centre for Astrophysics and Supercomputing, Swinburne University of Technology, PO Box 218, Hawthorn, VIC 3122, Australia \\ ${ }^{4}$ SKA SA, 3rd Floor, The Park, Park Road, Pinelands 7405, South Africa \\ ${ }^{5}$ Department of Physics and Electronics, Rhodes University, PO Box 94, Grahamstown 6140, South Africa \\ ${ }^{6}$ NVIDIA Corporation, 2701 San Tomas Expressway, Santa Clara, CA 95050, USA \\ ${ }^{7}$ California Institute of Technology, 1200 E California Blvd, Pasadena, CA 91125, USA \\ ${ }^{8}$ Department of Physics and Astronomy, University of New Mexico, Albuquerque, NM 87131, USA \\ ${ }^{9}$ Bradley Department of Electrical and Computer Engineering, Virginia Tech, Blacksburg, VA 24061, USA \\ ${ }^{10}$ Jet Propulsion Laboratory, California Institute of Technology, 4800 Oak Grove Dr., Pasadena, CA 91106, USA \\ ${ }^{11}$ National Radio Astronomy Observatory, PO Box O, Socorro, NM 87801, USA
}

Accepted 2018 May 9. Received 2018 May 8; in original form 2017 September 26

\begin{abstract}
The Large-aperture Experiment to Detect the Dark Age (LEDA) was designed to detect the predicted $\mathrm{O}(100) \mathrm{mK}$ sky-averaged absorption of the cosmic microwave background by hydrogen in the neutral pre- and intergalactic medium just after the cosmological Dark Age. The spectral signature would be associated with emergence of a diffuse Ly $\alpha$ background from starlight during 'Cosmic Dawn'. Recently, Bowman et al. have reported detection of this predicted absorption feature, with an unexpectedly large amplitude of $530 \mathrm{mK}$, centred at $78 \mathrm{MHz}$. Verification of this result by an independent experiment, such as LEDA, is pressing. In this paper, we detail design and characterization of the LEDA radiometer systems, and a first-generation pipeline that instantiates a signal path model. Sited at the Owens Valley Radio Observatory Long Wavelength Array, LEDA systems include the station correlator, five wellseparated redundant dual polarization radiometers and back-end electronics. The radiometers deliver a $30-85 \mathrm{MHz}$ band $(16<z<34)$ and operate as part of the larger interferometric array, for purposes ultimately of in situ calibration. Here, we report on the LEDA system design, calibration approach, and progress in characterization as of 2016 January. The LEDA systems are currently being modified to improve performance near $78 \mathrm{MHz}$ in order to verify the purported absorption feature.
\end{abstract}

Key words: instrumentation: detectors - telescopes - dark ages, reionization, first starscosmology: observations.

\section{INTRODUCTION}

Cosmic Dawn is a cosmological epoch extending between the buildup of the very first population of stars $\sim 100$ Myr after the big bang $(z \sim 30$, Bromm \& Yoshida 2011; Greif 2015; Hirano $\&$ Bromm 2017), followed by corresponding generations of black holes (e.g. Becerra et al. 2015; Smith, Bromm \& Loeb 2017; Smidt et al. 2017), to the onset of widespread reionization of the intergalactic medium (IGM) $\sim 500 \mathrm{Myr}$ after the big bang $(z \sim 10$, Robertson et al. 2015). This is one of the most interesting and least understood epochs in the history of the Universe (for a recent review, see e.g. 
Barkana 2016; Haiman 2016). Cosmic Dawn is marked by the rise of the earliest populations of sources (stars and black holes), rapid evolution of radiation fields, and the onset of metal enrichment (Wise et al. 2014; Safranek-Shrader et al. 2016).

Recently, Bowman et al. (2018) reported detection of the skyaveraged spectral signature of the $21-\mathrm{cm}$ ground-state transition of neutral Hydrogen (HI), placing Cosmic Dawn at redshifts $20>\mathrm{z}>15$. This signal, predicted by Shaver et al. (1999), is sensitive to both cosmological and astrophysical processes in the early Universe; as such, it is an excellent probe of the physics between the CMB decoupling and the end of the epoch of reionization. Indeed, if verified, the Bowman et al. (2018) result would constitute the earliest detection of the thermal footprint of the first stars (Greenhill 2018).

Specifically, Bowman et al. (2018) report detection of an $\sim 530 \mathrm{mK}$ absorption feature, centred at $\sim 78.1 \mathrm{MHz}$, with width $\sim 18.7 \mathrm{MHz}$, using a relatively simple - yet exquisitely calibrated - dipole antenna and radiometer system known as the Experiment to Detect the Global EoR Step (EDGES, Rogers \& Bowman 2012; Monsalve et al. 2017). The amplitude of this absorption feature is, remarkably, 2-3 times higher than that expected with the most optimistic models (Pritchard \& Loeb 2010; Fialkov, Barkana \& Visbal 2014; Fialkov \& Loeb 2016; Cohen et al. 2017). Also at odds with existing models, the feature is flat-bottomed, as opposed to Gaussian-like. The Bowman et al. (2018) result suggests gas temperatures during Cosmic Dawn were far cooler than previously predicted, and could even point toward interaction between baryons and dark-matter particles (Barkana 2018). An alternative explanation is that there was more radiation than expected, such as a significant contribution from an extragalactic background (Dowell \& Taylor 2018).

Nevertheless, some concerns remain that the purported Cosmic Dawn signal could in fact be an artefact, due to an unmodelled periodic instrumental feature, for example Hills et al. (2018). If verified, the Bowman et al. (2018) result places virtually the first observational constraints on Cosmic Dawn models. In comparison, the relatively more explored Epoch of Reionization (EoR; $z \sim 6$ $10)$, is somewhat constrained by (i) the integrated optical depth of Thomson scattering of cosmic microwave background (CMB) radiation (Planck Collaboration et al. 2016), (ii) the high-redshift galaxy ultraviolet (UV) luminosity function probed out to redshift of $z \sim 10$ (Bouwens et al. 2015; Atek et al. 2015), (iii) detection of dusty galaxies at redshifts out to $z \sim 10$ (Bouwens et al. 2016; Laporte et al. 2017), and (iv) supermassive black holes at $z \sim 7$ (Mortlock et al. 2011; Wu et al. 2015).

Cosmic Dawn is unique in terms of the astrophysical processes and sources that played roles. In contrast to the EoR, which was likely populated by a 'mature' population of galaxies residing in $\sim 10^{8.5}-10^{10} \mathrm{M}_{\odot}$ haloes (Mesinger, Greig \& Sobacchi 2016) and producing copious ionizing radiation, Cosmic Dawn was populated by pockets of intense star formation hosted in dark matter haloes of $\sim 10^{6}-10^{8} \mathrm{M}_{\odot}$, which were less efficient in ionizing their surroundings. Sources of X-rays, Ly $\alpha$, and Lyman-Werner (LW, 11.2$13.6 \mathrm{eV}$ ) radiation, on the other hand, played major roles during this epoch (e.g. Barkana 2016, and references therein), and direct study of this epoch is anticipated to deliver new knowledge about early stellar populations and to constrain formation scenarios for supermassive black holes (complementary to study of the EoR).

The preponderance of $\mathrm{HI}$ in the diffuse pre- and IGM (P/IGM) during Cosmic Dawn, and the sensitivity of the transition to radiative backgrounds produced by early stars and black holes makes the 21$\mathrm{cm}$ line a unique tracer of the early Universe. To date, the main focus of radio instruments undertaking '21-cm cosmology' (Pritchard \& Loeb 2010), has been detection of the of EoR power spectrum (i.e. large-scale spatial fluctuations). The Giant Meter-wave Radio Telescope (Paciga et al. 2013), the Precision Array for Probing the Epoch of Reionization (Ali et al. 2015; Pober et al. 2015), the Low Frequency Array (Patil et al. 2017), and the Murchison Widefield Array (Beardsley et al. 2016; Ewall-Wice et al. 2016) have all placed upper limits on the amplitude of the EoR power spectrum. The upcoming Hydrogen Epoch of Reionization Array (DeBoer et al. 2017), and the Square Kilometre Array telescope (Koopmans et al. 2015), also seek to constrain the EoR power spectrum.

Several experiments have been deployed in an attempt to measure the global 21-cm EoR signal. The first constraint on the global 21-cm EoR signal was provided by EDGES (Bowman, Rogers \& Hewitt 2008; Rogers \& Bowman 2012), which excluded reionization more rapid than $\Delta z>0.06$ with 95 per cent confidence. The Broadband Instrument for Global HydrOgen ReioNisation Signal (Sokolowski et al. 2015), and the Shaped Antenna measurement of the background RAdio Spectrum (SARAS, Patra et al. 2013; Singh et al. 2017) also target the global EoR signal, where results from the latter exclude at 68-95 per cent confidence some parameter combinations that correspond to late heating by X-rays in tandem with rapid reionization.

EDGES is one of several experiments designed to detect the global 21-cm Cosmic Dawn signal. The SARAS 2 experiment (Singh et al. 2018), SCI-HI (Sonda Cosmológica de las Islas para la Detección de Hidrógeno Neutro, Voytek et al. 2014), and the related Probing Radio Intensity at high $z$ from Marion (PRIZM) experiment, follow similar methodology and instrumentation approaches. The Dark Ages Radio Explorer concept proposes a satellite-based radiometer in lunar orbit, where earth occultation and absence of ionospheric effects are favourable (Datta et al. 2014; Burns et al. 2017).

Here, we detail the Large-aperture Experiment to Detect the Dark Age instrument (LEDA, see also Greenhill \& Bernardi 2012; Bernardi et al. 2016), which observes at frequencies between the HF $(3-30 \mathrm{MHz})$ and FM $(88-108 \mathrm{MHz})$ radio broadcast bands $(30<v<88 \mathrm{MHz}, 16<z<34)$. LEDA is unique in its embedding of radiometers in a densely interferometric array to enable calibration of radiometric data (in part) with observations of celestial sources (Section 8.1) and to create a ready path for exploration of power spectra estimation for Cosmic Dawn. In this paper, we present the design and characterization of the radiometry system for LEDA.

The paper is organized as follows. In Section 2, we provide a broad overview of the physics of Cosmic Dawn, details of the expected $21-\mathrm{cm}$ signal, and outline of experimental requirements needed to observe the Cosmic Dawn signal. We also point out astrophysical scenarios that can be detected or ruled out by LEDA. In Section 3, we set the stage discussing the site and the architecture of the telescope. LEDA radiometers are discussed in Section 4. Calibration is discussed in Section 5. In Section 6, we characterize the instrument, including gain linearity, reflection and transmission coefficients, receiver temperature, noise diode thermal stability, and temporal stability. Results, including absolute calibration, RFI occupancy, spectral index measurements, and comparison to extant sky models appear in Section 7. Discussion follows in Section 8.

\section{SCIENCE DRIVER}

The 21-cm line is a tracer of $\mathrm{HI}$ at all stages of cosmic evolution. Before the end of EoR, the signal is mainly produced by the inter- 


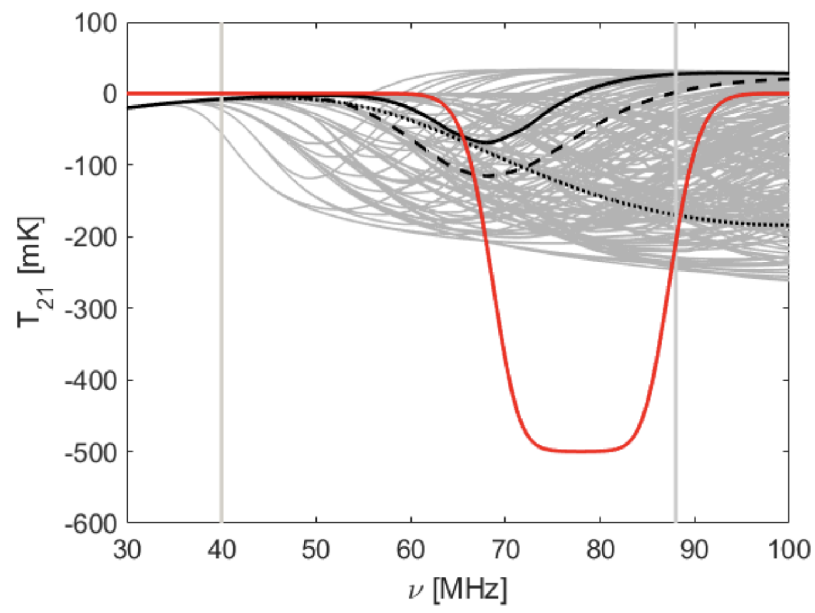

Figure 1. Spectra for variations in model parameters taken from Cohen et al. (2017), with purported EDGES detection from Bowman et al. (2018) in red. The heavy black traces show three particular scenarios with strong (solid), intermediate (dashed), and weak (dotted) signatures in the LEDA observing band (delineated by vertical solid lines).

galactic neutral medium; at lower redshifts, galactic HI dominates. The signal is sensitive to both cosmological and astrophysical processes, and as such, is arguably the best probe of the Universe at the intermediate-redshift range between the CMB decoupling and the end of reionization.

The observed differential brightness temperature relative to the $\mathrm{CMB}$ is

$T_{21} \approx 27 x_{\mathrm{HI}} \sqrt{\frac{1+z}{10}}\left(\frac{T_{\mathrm{S}}-T_{\mathrm{CMB}}}{T_{\mathrm{S}}}\right)[\mathrm{mK}]$,

where we have ignored terms of $\mathcal{O}(\delta)$, with $\delta$ being spatial density fluctuations. In equation (1), $x_{\mathrm{HI}}$ is the $\mathrm{HI}$ fraction, $T_{\mathrm{CMB}}$ is the $\mathrm{CMB}$ temperature, and $T_{\mathrm{S}}$ is the spin temperature defined as

$T_{\mathrm{S}}^{-1}=\frac{T_{\mathrm{CMB}}^{-1}+x_{\alpha} T_{\mathrm{c}}^{-1}+x_{\mathrm{c}} T_{\mathrm{K}}^{-1}}{1+x_{\alpha}+x_{\mathrm{c}}}$

where $T_{\mathrm{K}}$ and $T_{\mathrm{c}}$ are kinetic and collisional temperatures, and $x_{\alpha}$ and $x_{c}$ are Ly $\alpha$ and collisional coupling factors, respectively. Evolution of $T_{\mathrm{S}}$ with redshift is complex and depends on the intensity of the Ly $\alpha$ radiative background through the Wouthuysen-Field (WF) effect (Wouthuysen 1952; Field 1958) which sets $x_{\alpha}$, and on the thermal history of the Universe via $T_{\mathrm{K}}, T_{\mathrm{c}}$ (with $T_{\mathrm{c}} \approx T_{\mathrm{K}}$ ), and $x_{\mathrm{c}}$. The temperature $T_{21}$ may be positive or negative depending on the sign of $\left(T_{\mathrm{S}}-T_{\mathrm{CMB}}\right)$. For instance, when the transition is coupled to the gas temperature and the P/IGM is colder than the CMB, $T_{\mathrm{S}}<T_{\mathrm{CMB}}$ and the signal is seen in absorption $\left(T_{21}<0\right)$. When the gas is hotter than the $\mathrm{CMB}, T_{\mathrm{S}}>T_{\mathrm{CMB}}$ and the signal is seen in emission $\left(T_{21}>0\right)$.

For any particular scenario of structure and star formation, the evolution of $T_{21}$ can be used as a 'cosmic clock' that tracks the evolution of the Universe. In what follows, we focus on the zero mode of the signal from equation (1), a.k.a., the global signal. ${ }^{1}$ The predominant feature of the signal (e.g. the black curve in Fig. 1 which is a model with a strong signature in the LEDA observing band) is a Gaussian-like absorption trough signifying sufficient Ly $\alpha$

\footnotetext{
${ }^{1}$ Higher order modes are linked to spatial fluctuations and are outside of the scope of this paper (for more details see Barkana 2016, and references therein).
}

coupling and a cold diffuse medium. The centroid and amplitude of the trough depend directly on the balance between the processes of star formation and P/IGM heating. In particular, the low-frequency maximum (located at $v=46 \mathrm{MHz}$ for the black curve in Fig. 1) denotes the onset of star formation giving rise to the Ly $\alpha$ background. These photons coupled $T_{\mathrm{S}}$ to $T_{\mathrm{K}}$ via the WF effect, creating an absorption signature because at that epoch gas was colder than the CMB. The strongest absorption (at $v=68 \mathrm{MHz}$ for the black curve in Fig. 1) marks approximately the moment at which the IGM has reached its minimum temperature and a growing X-ray background due to compact sources becomes significant. As cosmic heating progresses, contrast between $T_{\mathrm{S}}$ and $T_{\mathrm{CMB}}$ decreases until the moment when the gas reaches the temperature of the background radiation, and the signal vanishes (at $v=87 \mathrm{MHz}$ for the black curve in Fig. 1). If this happens prior to reionization by UV sources, the signal will appear in emission for lower $z$.

The 21-cm spectra for an ensemble of astrophysical model parameters combinations $\left(M_{\min }, f_{*}, L_{\mathrm{X}}, \mathrm{X}\right.$-ray spectral energy distribution (SED), and the total CMB optical depth $\tau$ ) permitted by extant data and theoretical studies exhibit a large scatter as is shown in Fig. 1; the recent Bowman et al. (2018) result is overlaid in red. The amplitude of the CD trough varies between 25 and $240 \mathrm{mK}$, with the absorption trough located between $40<v<120 \mathrm{MHz}$ (Cohen et al. 2017). The Bowman et al. (2018) result is inconsistent with these models, exhibiting a much larger amplitude of $530 \mathrm{mK}$. Given this discrepancy, coupled with some outstanding concerns that the signal is an artefact (Hills et al. 2018), and that the result is yet to be verified, we do not as of yet rule these scenarios out.

For a large fraction of physically motivated models, and also for the purported Bowman et al. (2018) signal, the absorption minimum falls within the LEDA observing band, and, thus, could be detected by the instrument. This is discussed further in the next section.

\subsection{Observational prospects}

Radiometric detection requires separation of foreground signals and the (background) $21-\mathrm{cm}$ signal. The diffuse and continuum foreground sources are known to be spectrally smooth; that is, they exhibit power-law spectra over the $30-88 \mathrm{MHz}$ band. As such, they are separable from the background signal, which is expected to manifest as an absorption trough. Pritchard \& Loeb (2010) showed a basic demonstration of concept, by convolving a Global Sky Model (GSM, de Oliveira-Costa et al. 2008) with an analytical model for a simple dipole antenna to form simulated measurements; this approach is followed by several Cosmic Dawn experiments (Voytek et al. 2014; Bowman et al. 2018; Singh et al. 2018), including LEDA.

The spectral smoothness of foregrounds allows retrieval of 21$\mathrm{cm}$ features by modelling the brightness temperature of the foreground, $T_{\text {fg }}$, with a low-order log-polynomial. Harker et al. (2012) expounded on this by including instrumental effects; Bernardi, McQuinn \& Greenhill (2015) showed that the angular structure and frequency dependence of a more realistic broad-band dipole (modelled on the design of the Long Wavelength Array) increases the required polynomial order but not necessarily so much so as to confound detection. For this approach to work, any spectral structure introduced by the measurement apparatus must be accounted for and calibrated out. For this reason, zero-mode radiometer experiments have preferred simple, low-gain dipole antennas over high-gain single dishes that exhibit more complex gain patterns (Bernardi et al. 2015; Mozdzen et al. 2016). We discuss the approach of LEDA to calibration in Section 5. 
Significance of the detection by LEDA is determined by tiny deviations in the shape of the actual sky temperature from the smooth foreground curve in the LEDA band. To estimate which part of the astrophysical parameter space is actually targeted by LEDA, we use the signal-to-noise ratio $(\mathrm{S} / \mathrm{N})$ defined as

$\mathrm{S} / \mathrm{N}^{2}=\sum_{i} \frac{\left(T_{\text {sky }}-\tilde{T}_{\mathrm{fg}}\right)^{2}}{\sigma_{\mathrm{i}}^{2}}$

where the sum is over frequency channels in the LEDA band, the mock data are defined as $T_{\text {sky }}=T_{\mathrm{fg}}+T_{\text {cosm }}$ and the foreground signal is modelled as a seventh-order polynomial in logv (Bernardi et al. 2016). We fit out the foreground component by calculating $\tilde{T}_{\text {fg }}$ as a best fit to the mock data of the shape provided by Bernardi et al. (2016). The residual signal is then compared to the rms noise given by the radiometer equation

$\sigma \approx 2.6 \times \frac{T_{\text {sys }}}{\sqrt{\Delta v t}}$

where $\Delta v$ is the bandwidth over which the signal is measured, and $t$ is the integration time, and we assumed that the system temperature, $T_{\text {sys }}$, is dominated by the temperature of the sky. The factor 2.6 is an approximation that takes into account thermal uncertainties after calibration. (Here, we assumed hot and cold reference diode temperatures of 6500 and $1000 \mathrm{~K}$, as is explained in detail in Section 5 , equation 18.) Thus, for a sky temperature of $2000 \mathrm{~K}$ and using $\Delta v=1 \mathrm{MHz}$, a $5 \sigma$ detection of the Bowman et al. (2018) feature could be made in under 45 min of observation with LEDA.

Alternatively, one may calculate $\mathrm{S} / \mathrm{N}$ for each of the $\sim 200$ models shown in Fig. 1, assuming $\Delta v=1 \mathrm{MHz}$ and integration time of 1000 h. Out of $\sim 200$ different astrophysical scenarios (Fig. 1), the models with the highest $\mathrm{S} / \mathrm{N}$ are those with strongest variation within the LEDA band. These models typically share high star formation efficiency (often in low-mass haloes) and high X-ray efficiency, which suggests that LEDA should have considerable leverage in constraining (i) star formation during Cosmic Dawn, and in particular the roles of small haloes, and (ii) the timing of $\mathrm{X}$-ray heating and properties of high-redshift X-ray sources (e.g. $\mathrm{X}$-ray background, $\mathrm{XRB}$, and mini-quasars). The model with the highest $\mathrm{S} / \mathrm{N}$ ratio $=9.2$ (heavy solid curve in Fig. 1) shows both a strong absorption and an early emission signal within the LEDA band. These features are hard to mimic with smooth foregrounds. The underlying astrophysical model assumes high star formation efficiency of $f_{*}=50$ per cent in heavy haloes above circular velocity of $35.5 \mathrm{~km} \mathrm{~s}^{-1}$ and a very luminous XRB population shining at the luminosity of $L_{\mathrm{X}}=15 \times 10^{41} \mathrm{erg} \mathrm{s}^{-1}$ per unit star formation rate in $\mathrm{M}_{\odot} \mathrm{yr}^{-1}$ (i.e. 50 times brighter than the low-redshift counterparts).

In another detectable scenario (heavy dashed curve) only the absorption trough is located within the LEDA band which makes the detection a bit more challenging. The underlying astrophysical model has moderate star formation efficiency, $f_{*}=5$ per cent, stars form via cooling of atomic hydrogen, $\mathrm{X}$-ray heating is due to XRB with $L_{\mathrm{X}}=2.4 \times 10^{41} \mathrm{erg} \mathrm{s}^{-1}$ per unit star formation rate in $\mathrm{M}_{\odot}$ $\mathrm{yr}^{-1}$, and the total CMB optical depth of $\tau=0.066$. The moderate star formation and heating result in a moderate $S / N=4$ in the LEDA band. Finally, in Fig. 1, we also show an astrophysical scenario that cannot be separated from the foregrounds, and, thus, is undetectable by LEDA ( $\mathrm{S} / \mathrm{N}=0.1$, dotted line in the figure). This case has low star formation efficiency, $f_{*}=0.5$ per cent, star formation via molecular hydrogen cooling subjected to strong LW feedback, and weak X-ray heating with $L_{\mathrm{X}}=0.03 \times 10^{41} \mathrm{erg} \mathrm{s}^{-1}$.

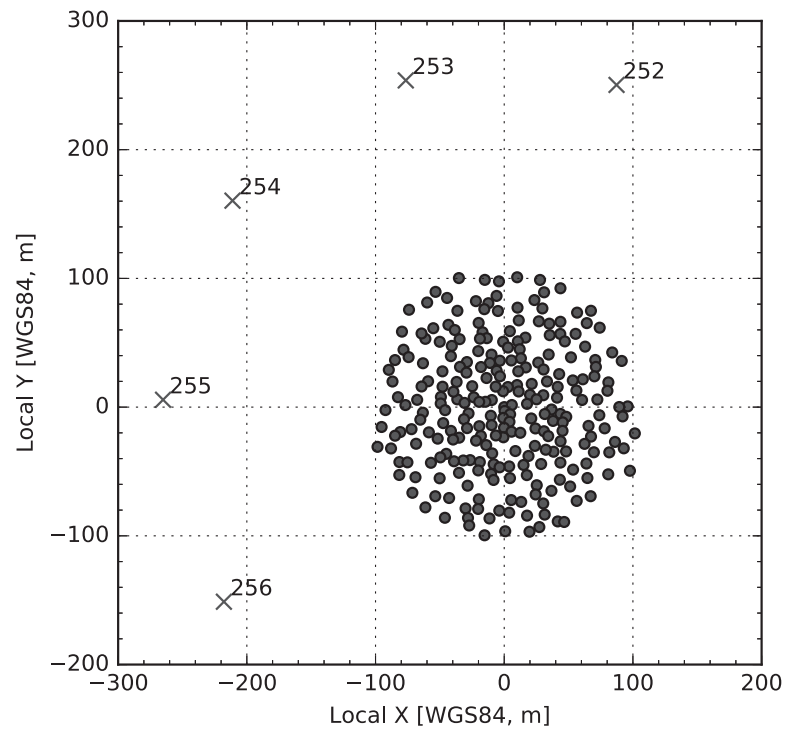

Figure 2. Antenna positions in the WGS84 coordinates relative to the mean. Crosses represent the outrigger antennas equipped with LEDA radiometric front ends. (Antennas installed at greater separations in 2015 are not shown.)

\section{INSTRUMENT OVERVIEW}

Motivated by limitations of single-antenna experiments, LEDA is a multi-antenna experiment, co-installed on the Long Wavelength Array stations at Owens Valley Radio Observatory (OVRO-LWA, $37.24^{\circ} \mathrm{N}, 118.28^{\circ} \mathrm{W}$ ), and the National Radio Astronomy Observatory in Socorro, New Mexico (LWA1, $34.07^{\circ} \mathrm{N}, 107.63^{\circ} \mathrm{W}$ ). Initial work, based at LWA1, is presented in (Schinzel et al., LWA Memo \#208); here we focus on work carried out at OVRO-LWA. Further details about the LWA systems may be found in Taylor et al. (2012) and Ellingson et al. (2013).

At both the LWA1 and OVRO-LWA stations, an additional five outrigger stands (Fig. 2) were installed and outfitted with the LEDA front-end receiver card (see Section 4). The LEDA outrigger stands, detailed further in Section 3.3, are placed at a distance from the core to minimize mutual coupling effects. Bernardi et al. (2015) argue that detection will require precise knowledge of the antenna radiation pattern, which may not be deliverable by electromagnetic (EM) simulation. Datta et al. (2014) are pessimistic that a detection may be made without an accurate model of the ionosphere. There is also concern that terrestrial radio frequency interference (RFI) could confound detection.

The OVRO-LWA core region consists of 251 stands within a $100-\mathrm{m}$ radius (Fig 2), roughly double the radius of LWA1. The OVRO-LWA station (Fig. 3) was built in 2013 and utilizes the same antenna design and analogue systems as LWA1, with a different digital system designed for wide-bandwidth cross-correlation (Kocz et al. 2015). An additional five outrigger stands (Fig 4) were installed and outfitted with the LEDA front-end receiver card - the characterization and design of which is the focus of this article. A more complete overview of OVRO-LWA may be found in Hallinan et al. (in preparation).

\subsection{Observational strategy}

OVRO-LWA allows for LEDA to monitor the ionosphere, characterize the foreground sky, and measure antenna gain patterns in situ, all while radiometric measurements are being taken. Gain patterns 


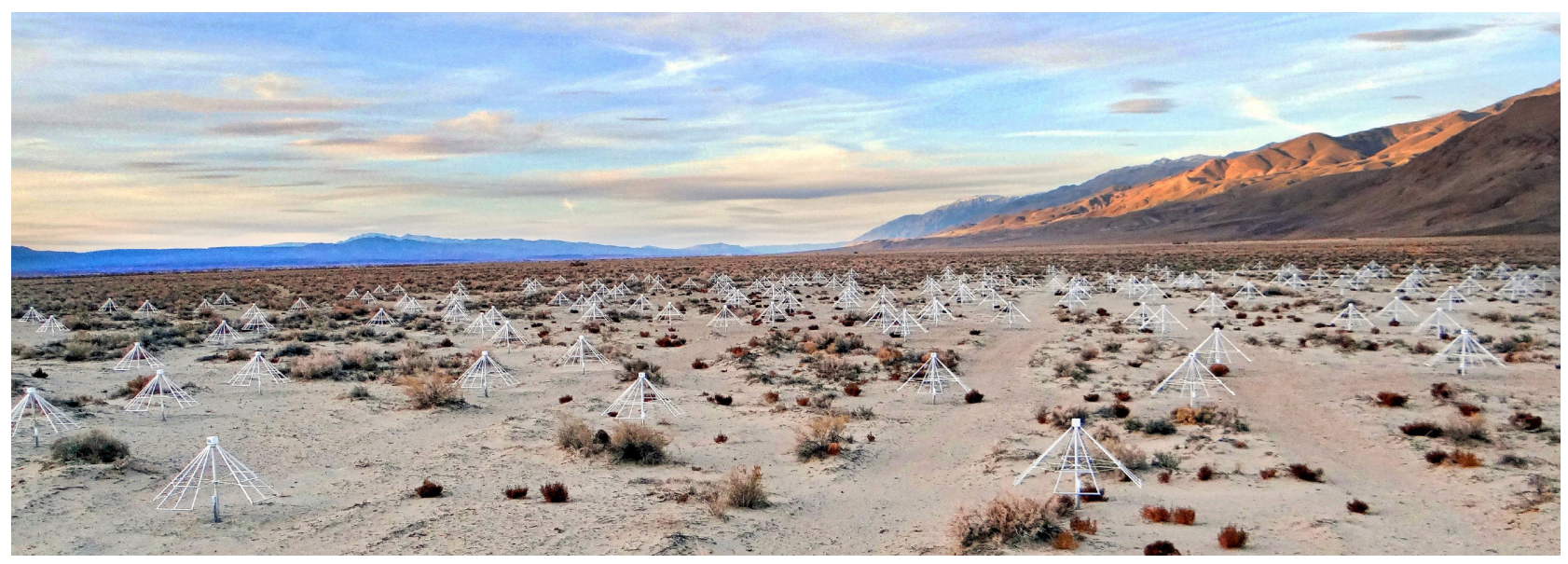

Figure 3. The core area of the Owens Valley LWA, looking north from the electronics shelter located at the edge. The dual-polarization dipoles are aligned north-south and east-west.

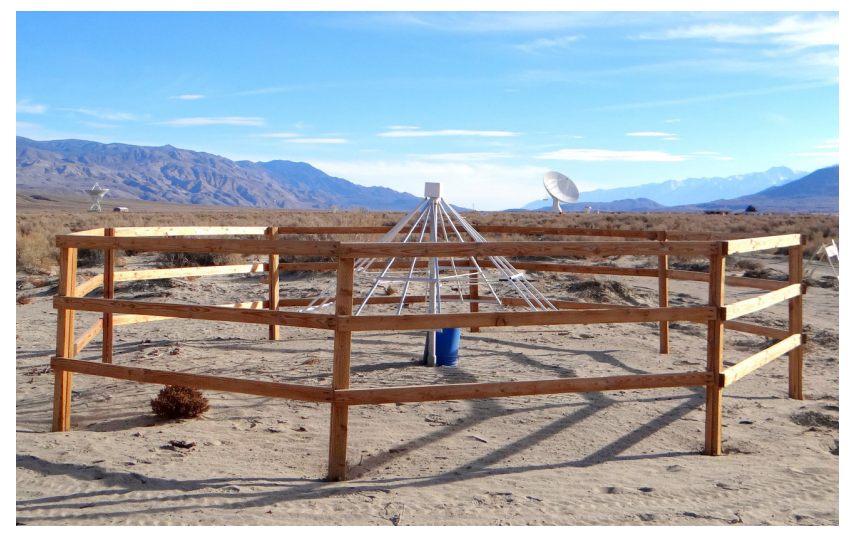

Figure 4. LEDA outrigger antenna stand (ID 256) at Owens Valley Radio Observatory, California, 2015 January.

are measured via cross-correlation of LEDA radiometer antennas against the dense core of OVRO-LWA. The OVRO-LWA array is capable of imaging the radio sky from horizon to horizon; by doing so, the position and apparent brightness of celestial sources can be monitored. By monitoring the passage of celestial sources, a beam model can be inferred; similarly, a model of ionospheric-induced refractive offsets may be formed by monitoring offsets in the apparent positions of sources.

The LEDA observational strategy is implemented to avoid complications that arise due to synchronization requirements between the noise diode switching and correlator integration. In order to derive gain patterns, once a week a 24-h interferometric observation of the sky is performed at a low duty cycle (a $10 \mathrm{~s}$ integration every $100 \mathrm{~s}$ ). These data are also used to form a model of the sky. During these observations, the radiometer antennas do not switch into diode states. In contrast, during radiometric observations the ionosphere is monitored using the core antennas and cross-correlations with the outriggers are discarded.

The primary LEDA observational window is during night-time hours over December-March. The Sun is a potential source of interference, and RFI is known to be more prevalent during daylight hours. Furthermore, at low frequencies, the galactic plane has a significant contribution to the overall system temperature of a ra- diometer, so it is desirable to observe when the galactic plane is low.

\subsection{LEDA correlator and spectrometer}

OVRO-LWA is operated as a radio interferometer, which requires the cross-correlation of all antenna pairs. Cross-correlation is an $O\left(N^{2}\right)$ operation, which is computationally challenging for the $N$ $=512$ inputs of OVRO-LWA. The cross-correlation of all 256 dual-polarization antenna pairs is performed by the LEDA correlator, detailed in Kocz et al. (2014, 2015). Briefly, the LEDA correlator is a, FX-style system where the data are channelized (by 'F-engines') before cross-correlation by the 'X-engine'. The F-engines run on ROACH-2 (Reconfigurable Open-Architecture Compute Hardware) field-programmable gate array boards from the Collaboration for Astronomy Signal Processing and Electronics Research (CASPER, Hickish et al. 2016). The F-engines are connected via $10 \mathrm{~Gb}$ Ethernet to compute servers running the xGPU cross-correlation X-engine code (Clark, Plante \& Greenhill 2013).

The firmware for the LEDA F-engine has been modified from that detailed in Kocz et al. (2015) to generate autocorrelation spectra at higher bit depth. The LEDA correlator requantizes the output of the polyphase filterbank (PFB) down to 4 bits, for data transport to the computer servers. The modified firmware generates autocorrelation spectra from the (18-bit) F-engine output before requantization, yielding higher dynamic range and lowering quantization-induced non-linearity compared to the 4-bit data stream. We refer to this system as the LEDA spectrometer.

All spectral data products presented here are from the LEDA spectrometer. Corresponding cross-correlation data from the LWA core were also recorded for ionospheric monitoring, but are not used further within this article. The spectrometer is implemented using a 4096-channel, 4-tap, Hamming-windowed PFB. The PFB provides $\sim 50 \mathrm{~dB}$ of isolation between neighbouring channels, which prevents leakage of narrowband RFI signals between channels. The digitizer is clocked at $196.608 \mathrm{MHz}$, resulting in a $24 \mathrm{kHz}$ channel bandwidth. The output of each PFB channel is squared and accumulated for $1 \mathrm{~s}$. An external pulse-per-second (PPS) signal is used to trigger each new accumulation. Accumulated data are read from the ROACH-2 board's Ethernet control interface. After every accumulation, data are timestamped and written to a hierarchical data format (HDF5) file. 


\subsection{Outrigger antennas}

The outrigger antennas (Fig. 4) are of the same design as the LWA cross-dipole antennas, detailed in Ellingson et al. (2013). Each dual-polarization antenna consists of four triangular 'blades' of length $1.4 \mathrm{~m}$, which form two pairs of orthogonal antennas (singlepolarization). The blades are attached to a central pole of height $1.5 \mathrm{~m}$, at the top of which is a weatherproof box for the FE. The two antenna pairs are oriented north-south and east-west. The blades are angled down to improve response at the horizon and beam symmetry. A wire-grid $3 \times 3-\mathrm{m}$ ground screen isolates the antennas from the earth ground, whose characteristics may change with moisture content.

The outrigger antennas are physically isolated from the core antennas and other metallic objects. Each antenna has a 3×3-m ground screen, and is protected from grazing cattle by a wooden fence. The antennas are connected to the shelter via buried lengths of LMR400 coaxial cable. These cables are fed up through the central pole of the antenna, through to the receiver.

\section{LEDA RADIOMETERS}

In this section, we provide further detail about a single radiometer system; in total, there are $5 \times 2$ complete radiometers within LEDA. A block diagram of a LEDA radiometer is given in Fig. 5 for a single polarization. As seen in the diagram, there are four main components: the antenna, front-end receiver, back-end analogue systems, and digital systems. Each dual-polarization antenna is connected directly at its terminals to the front-end electronics (FE). The FE converts the balanced antenna terminal pair to unbalanced $50 \Omega$ via a $4: 1$ balun, then amplifies and filters the signal, outputting the conditioned signal over a buried coaxial cable to the OVRO-LWA electronics shelter.

The entry bulkhead of the shelter connects the buried coaxial to an FM bandstop and lightning arrestor installed at the bulkhead; a length of coaxial cable connects the bulkhead to the back-end analogue systems (CRX).The CRX applies further amplification and signal filtering, in preparation for digitization. The CRX systems also provide power to the FE, via the coaxial cable lengths that connect the antenna to the shelter. The signal from the CRX is converted from $50 \Omega$ unbalanced to $100 \Omega$ balanced, and Category-7A Ethernet cables are used to transport the signals to the digitizer. Further details of these systems are given below.

\subsection{Deployment history}

The LEDA radiometer systems are under active development; iterative upgrades and improvements are made after each field deployment (Table 1). A brief historical summary of deployments is as follows. The LEDA correlator system achieved first light in 2013 August, using an early revision of the FE card (version 2.0) along with the standard LWA analogue receiver system (ARX), with spectra formed from 4-bit data sent to the correlator. In 2013 December, the correlator F-engine firmware was modified to add an independent autocorrelator spectrometer with higher bit depth. An updated version of the FE with added MS147 test ports (version 2.5) was installed in 2014 April. Due to concerns of potential crosstalk, a fully shielded switching controller (Section 4.3) was installed in 2014 November, and the LWA analogue receiver was replaced by a fully connectorized system in 2014 December. Major improvements were made to the FE over the course of 2015; the FE version 2.9 was deployed in 2016 January.
In this paper, we detail the system as installed at OVRO-LWA in 2016 January; details of the FE version 2.5 and LWA1 results are presented in Schinzel et al. (2018).

\subsection{Front-end receiver board}

The LEDA FE (Fig. 6) connects to the antenna terminals (Fig. 7) and applies first-stage signal amplification and conditioning. The FE is a two-sided, four-layer circuit board of dimensions of $11.5 \times 11.5 \mathrm{~cm}$, installed in the weatherproof box at the antenna's apex. The FE provides signal paths for both stand polarizations, one per side.

A differential $200 \Omega$ line connects each blade pair to a MiniCircuits ADT4-6T transformer (balun) that converts the balanced signal to $50 \Omega$ unbalanced. A four-throw switch (Mini-Circuits GSWA-4-30DR) allows for selection between the antenna path and two calibration reference paths (Section 4.2.3). The switch output then connects to the first-stage low noise amplifier (LNA, MiniCircuits Gali-74+, Table 2). A bandpass filter (Mini-Circuits BPFC45+) suppresses signals outside of $25-90 \mathrm{MHz}$, attenuating HF and FM RFI sources outside the band of interest. Mini-Circuits LAT attenuators are used between components to improve impedance matching.

Second-stage signal amplification is done after filtering, using two Mini-Circuits Gali-6+ amplifiers connected in cascade, each with $12.2 \mathrm{~dB}$ gain. While the Gali-74+ has a lower noise figure than the Gali-6+, the Gali-6+ was chosen for its flatter gain response over the LEDA band. Along the signal path, several buffering attenuators are installed to improve impedance match between components.

Table 2 shows the cascaded gain $\left(G_{\mathrm{rx}}\right)$ and receiver temperature $\left(T_{\mathrm{rx}}\right)$ after each component in the FE analogue path. The overall receiver temperate $T_{\mathrm{rx}}=432 \mathrm{~K}$, and the receiver gain $G_{\mathrm{rx}}=31.2 \mathrm{~dB}$. Note that these values are calculated from specifications provided in component data sheets; and actual measurements are provided in Section 6.

Losses before the first-stage LNA and the LNA's noise temperature dominate $T_{\text {rx }}$. The overall $T_{\text {sys }}$ of a LEDA radiometer is none the less dominated by the antenna temperature, which is $>1000 \mathrm{~K}$ across the LEDA band.

\subsubsection{DC power and state control}

The FE receives DC power via its SMA output jack. The RF signal and DC power are separated by an onboard bias tee. The switch state is controlled by changing the DC voltage supplied to the board, between $17 \mathrm{~V}$ (sky), $20 \mathrm{~V}$ (cold reference), and $23 \mathrm{~V}$ (hot reference). This allows FE state to be controlled remotely from the electronics shelter.

Many of the components on the board require $12 \mathrm{~V}$ or lower. Regulation to $12 \mathrm{~V}$ is conducted on an external board, which connects to the FE via a ribbon cable; the thermal load of the regulators would produce undesired thermal gradients if placed directly on the FE.

\subsubsection{Test ports}

The ability to measure reflection coefficients of components is essential for absolute calibration of the LEDA radiometers. To facilitate this, Hirose MS147 test ports have been added to the circuit. When an MS147 cable is connected to the test port, an internal mechanical switch within the test port reroutes the circuit to the MS147 cable. The MS147 ports are used to measure the reflection coefficients, $\Gamma_{\text {ant }}$ and $\Gamma_{\mathrm{rx}}$, as introduced in Section 5. 


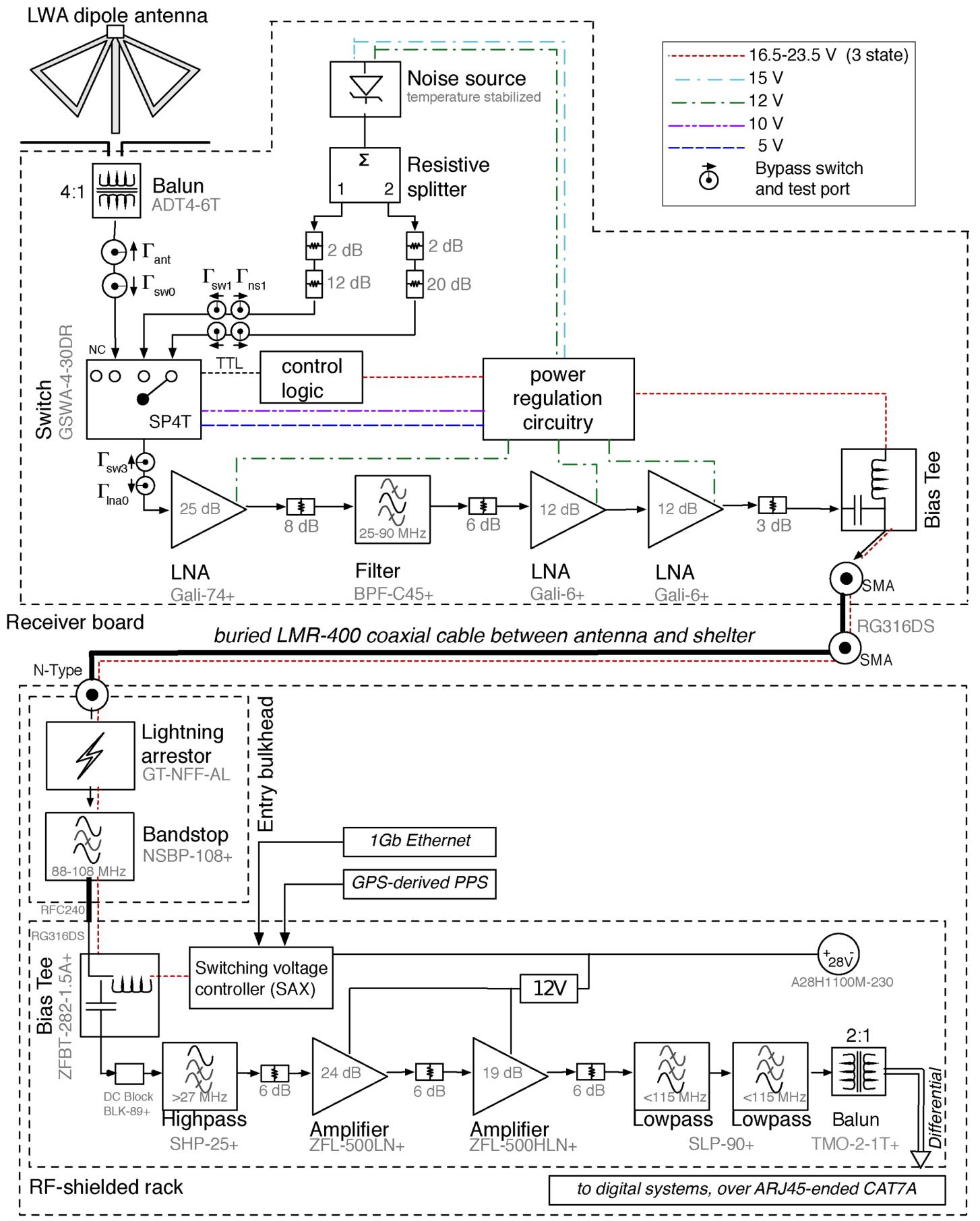

RF-shielded shelter

Figure 5. Block diagram of a single polarization radiometer analogue signal path. Components on a receiver board are shown in the dashed box in the upper half. (See also Fig. 6.) RF jacks (Hirose MS147) provide test ports for measurement of reflection coefficients. When in use, the through path is broken, and the network analyser looks in the direction indicated. Components in the central electronics shelter are in the lower half. Details of the temperature stabilized noise source are shown in Fig. 8. DC supply lines are in colour.

\subsubsection{Calibration subcircuit}

At LEDA frequencies, the intrinsic sky noise $(>1000 \mathrm{~K})$ is much greater than ambient temperature $(\sim 300 \mathrm{~K})$. As such, comparison to an ambient $50 \Omega$ load results in a large swing in LNA input power; this is undesirable for two reasons. First, digitizer dynamic range requirements are reduced, allowing more overhead to deal with radio interference. Secondly, large swings in power change which bits in the digitizer are being exercised, and are consequently more likely to be affected by non-linear quantization gain. Additionally, the S/N after applying three-state switching (equation 19) is improved by using stronger references. As such, the reference calibration states on the FE are provided by a noise diode-based subcircuit, with equivalent noise temperatures better matched to the sky temperature.

The calibration subcircuit is shown in Fig. 8. This circuit is based 
Table 1. Summary of radiometer system upgrades 2013-2016.

\begin{tabular}{|c|c|}
\hline mmyy & Deployment milestones \\
\hline 08/13 & First light $^{a}$ \\
\hline $12 / 13$ & $\begin{array}{l}\text { Embedded standalone 8-bit } \\
\text { spectrometers }^{\mathrm{b}}\end{array}$ \\
\hline $04 / 14$ & FE version 2.5: bandpass filtering \\
\hline $11 / 14$ & $\begin{array}{l}\text { Shielded programmable switching } \\
\text { controller }\end{array}$ \\
\hline $12 / 14$ & $\begin{array}{l}\text { Shielded, low crosstalk back-end } \\
\text { systems, high- } \\
\text { isolation standard for multichannel } \\
\text { cables }^{c}\end{array}$ \\
\hline $01 / 16$ & $\begin{array}{c}\text { FE version 2.9: improved impedance } \\
\text { matches, better } \\
\text { noise source stability, high RF } \\
\text { directivity }\end{array}$ \\
\hline
\end{tabular}

${ }^{a}$ FE version 2.0, unshielded $1 \mathrm{~Hz}$ switching controller, LWA analogue back end, and LEDA correlator.

${ }^{b}$ Phased out use of station correlator for precision radiometric data. ${ }^{c}$ Adoption of Bel-Stewart ARJ45 differential cable-end standard for RF over twisted pair between analogue receivers and correlator digital samplers.

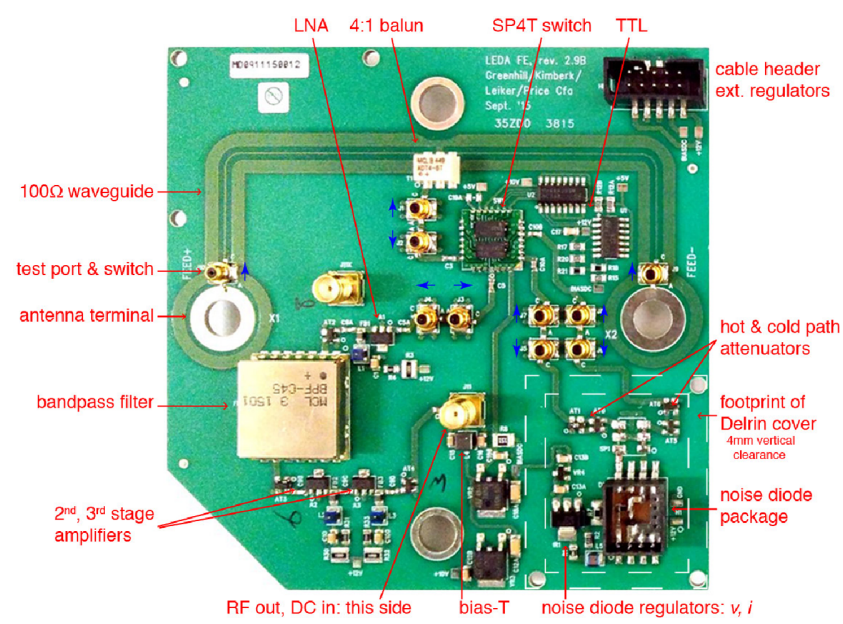

Figure 6. Photograph of the LEDA v2.9 FE, with key components labelled. The Delrin cover and heater has been removed so that the noise diode is visible.

on a NoiseWave NC501-12/SM noise diode package, which we have modified to improve its stability. The top plastic cover of the NC501 was removed and replaced by a DN505 heater seated upon an aluminium block. The DN505 is set to maintain a constant temperature of $60^{\circ} \mathrm{C}$, to mitigate ambient temperature variations. The circuit includes a constant voltage regulator (LM3480) and constant current regulator (LT3092) to ensure the noise diode receives a stable DC supply.

A resistive splitter is used to provide two calibration paths, upon which we place different attenuators to yield 'hot' and 'cold' references. Although a resistive splitter gives $3 \mathrm{~dB}$ of loss as compared to an ideal power divider, we found that thermal stability - the change in noise power output as a function of ambient temperature - was notably worse when a power divider was used; see Section 6.5 for measurement details.

\subsection{Analogue back end}

Second-stage signal conditioning for the LEDA radiometers is performed using a connectorized analogue receiver chain (CRX, Fig. 9). While the station is outfitted with an LWA 512-input secondstage ARX (Ellingson et al. 2013), used to amplify and filter antenna signals prior to correlation, this system was not suitable for precision radiometry: the ARX demonstrated crosstalk between neighbouring channels, pickup of radiation emitted by the densely packed signal paths inside the shielded analogue rack, and reflection along the signal path that affected the noise floor.

\subsubsection{Connectorized receiver system}

The first two components of the CRX are situated at the RF-shielded shelter's entry bulkhead: a Lightning arrestor (PolyPhaser GT-NFFAL) and an FM bandstop filter (Mini-Circuits NBSP-108+). An RG316-DS coaxial cable connects the bulkhead components to the rest of the CRX, which is located in an RF-shielded rack. A bias tee (Mini-Circuits ZFBT-282-1.5A+) supplies DC voltage back to the antenna, while blocking DC on the output port. A high-pass filter (Mini-Circuits SHP-50+) filters out HF interference, this is connected to a Mini-Circuits ZFL-500LN+ amplifier $(G=24 \mathrm{~dB})$. A further $19 \mathrm{~dB}$ of amplification is provided by a ZFL-500HLN+ amplifier, which is optimized for higher power than the preceding $500 \mathrm{LN}+$ model. Final-stage filtering of the FM band and above is then provided by two Mini-Circuits SLP-90+ filters in cascade. For improved impedance matching, Mini-Circuits VAT-6+ $6 \mathrm{~dB}$ attenuators are placed before, between, and after the two amplifiers.

\subsubsection{Switching assembly}

In order to select between sky and reference diode states on the FE, different DC power levels are supplied via the CRX bias tees. The DC power supplied to the CRX bias tees is controlled by the switching assembly (SAX), shown in Fig. 5 as the receiver board voltage controller. The SAX system consists of a custom voltage regulation circuit controlled by a Rabbit 3000 Microprocessor via a wired Ethernet connection. The SAX accepts DC input power from a $28 \mathrm{~V}, 11 \mathrm{~A}$ Acopian Gold linear supply (A28H1100-230), and outputs power at 17,20 , and $23 \mathrm{~V}$, as required by the FE. Tunable potentiometers may be used to adjust the output power, to account for power drop over the coaxial cables (1-1.5 V).

The SAX accepts a PPS signal, which may be used to trigger state changes on the FE. Alternatively, FE state may be controlled manually, by issuing commands to the Rabbit microprocessor over Ethernet. As the SAX is located in the analogue rack, it is encased in an RF-tight box, to shield any microprocessor-generated RF power from the analogue systems.

\subsection{Inter-rack signal transport}

The digital and analogue systems are housed in separate RFshielded racks that share a common bulkhead wall. Consistent with the LWA engineering model, CRX signals in the analogue rack are converted from unbalanced $50 \Omega$ to balanced $100 \Omega$ for transmission to the digital rack on Category-7a (CAT7A) Ethernet cable. A custom-made balun module converts every $4 \times$ SMA inputs into a single Belfuse ARJ45-ended output (one conductor pair per RF signal path). Custom RF-tight, CAT-7A CONEC feedthroughs route signals through bulkhead plates. 

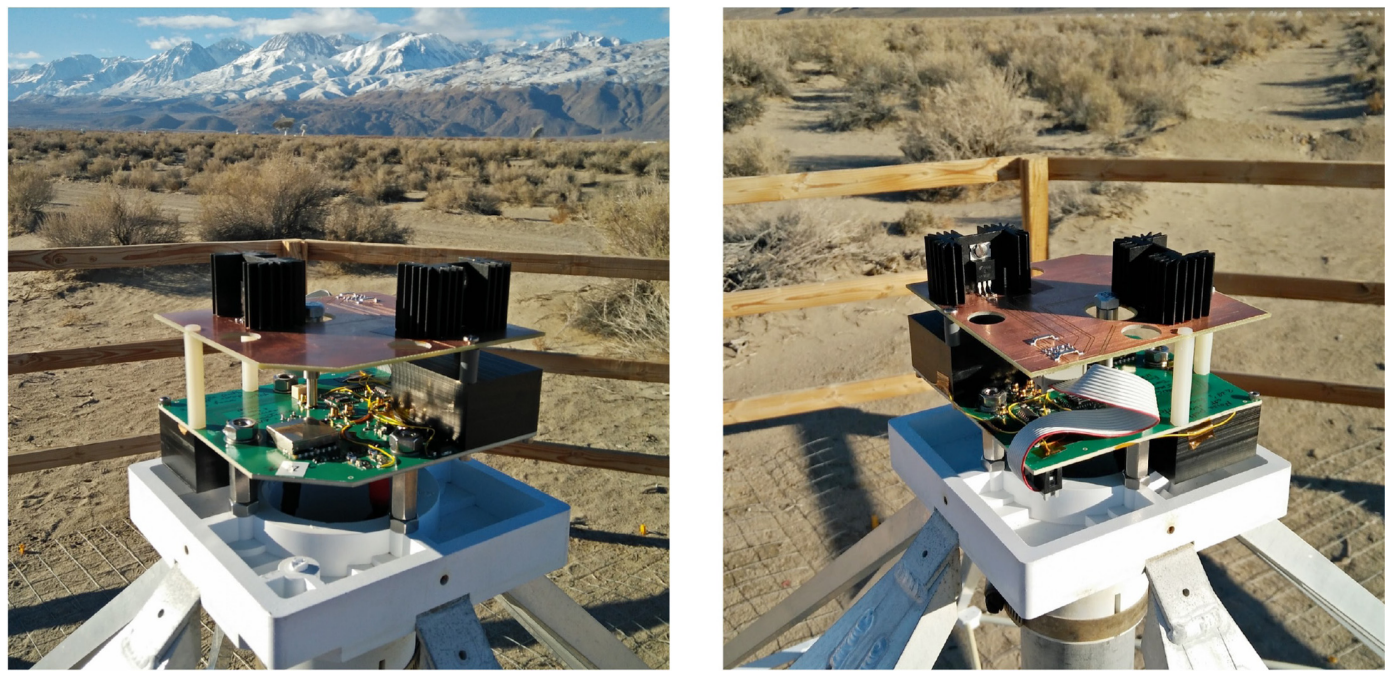

Figure 7. LEDA v2.9 radiometer FE, as installed on an OVRO-LWA outrigger antenna, as seen from the north-west (left) and south-east (right). As for LWA hardware, the threaded posts serve as antenna terminals. Upward facing electronics on the FE card serves the east-west dipole. Downward facing electronics serve the north-south dipole. For this photo, the protective plastic cover has been removed.

Table 2. Cascaded FE noise temperatures at $50 \mathrm{MHz}$.

\begin{tabular}{|c|c|c|c|c|c|c|c|}
\hline \multirow[t]{2}{*}{ Component } & \multicolumn{2}{|c|}{ Noise figure } & \multicolumn{2}{|c|}{ Gain $\left(S_{21}\right)$} & \multirow{2}{*}{$\begin{array}{c}T_{\text {noise }} \\
(\mathrm{K})\end{array}$} & \multirow{2}{*}{$\begin{array}{l}T_{\mathrm{rx}} \\
(\mathrm{K})\end{array}$} & \multirow{2}{*}{$\begin{array}{c}G_{\mathrm{rx}} \\
(\mathrm{dB})\end{array}$} \\
\hline & $(\mathrm{dB})$ & $\left(\mathrm{dB} \mathrm{K}^{-1}\right)$ & $(\mathrm{dB})$ & $\left(\mathrm{dB} \mathrm{K}^{-1}\right)$ & & & \\
\hline GSWA-4-30DR ${ }^{\mathrm{a}}$ & - & - & -0.7 & - & 53.1 & 54.6 & -0.7 \\
\hline Gali- $74^{\mathrm{b}, \mathrm{c}}$ & 2.64 & -0.0052 & 24.8 & -0.0026 & 249.4 & 373.7 & 24.1 \\
\hline LAT8 & - & - & -8.1 & - & 1613.6 & 380.5 & 16.0 \\
\hline BPF-C45+ & - & - & -0.4 & - & 25.8 & 381.2 & 15.7 \\
\hline LAT6 & - & - & -6.2 & - & 950.5 & 409.1 & 9.4 \\
\hline Gali-6 ${ }^{\mathrm{b}, \mathrm{c}}$ & 4.27 & -0.0087 & 13.0 & -0.0015 & 470.5 & 428.5 & 22.5 \\
\hline
\end{tabular}

${ }^{a}$ Manufacturer specifications at $64 \mathrm{MHz}$.

${ }^{b}$ Manufacturer specified gain for $T=25 \mathrm{C}, 50 \Omega$ source and load, and $i_{\mathrm{cc}}=65 \mathrm{~mA}$ (Gali-74) or $i_{\mathrm{cc}}=70 \mathrm{~mA}(\mathrm{Gali}-6)$. Measured currents are 61 and $68 \mathrm{~mA}$, respectively. Component operating temperatures are $\sim 40^{\circ} \mathrm{C}$ for an ambient temperature of $25^{\circ} \mathrm{C}$.

${ }^{c}$ Manufacturer specified noise figure for test conditions in note (b).
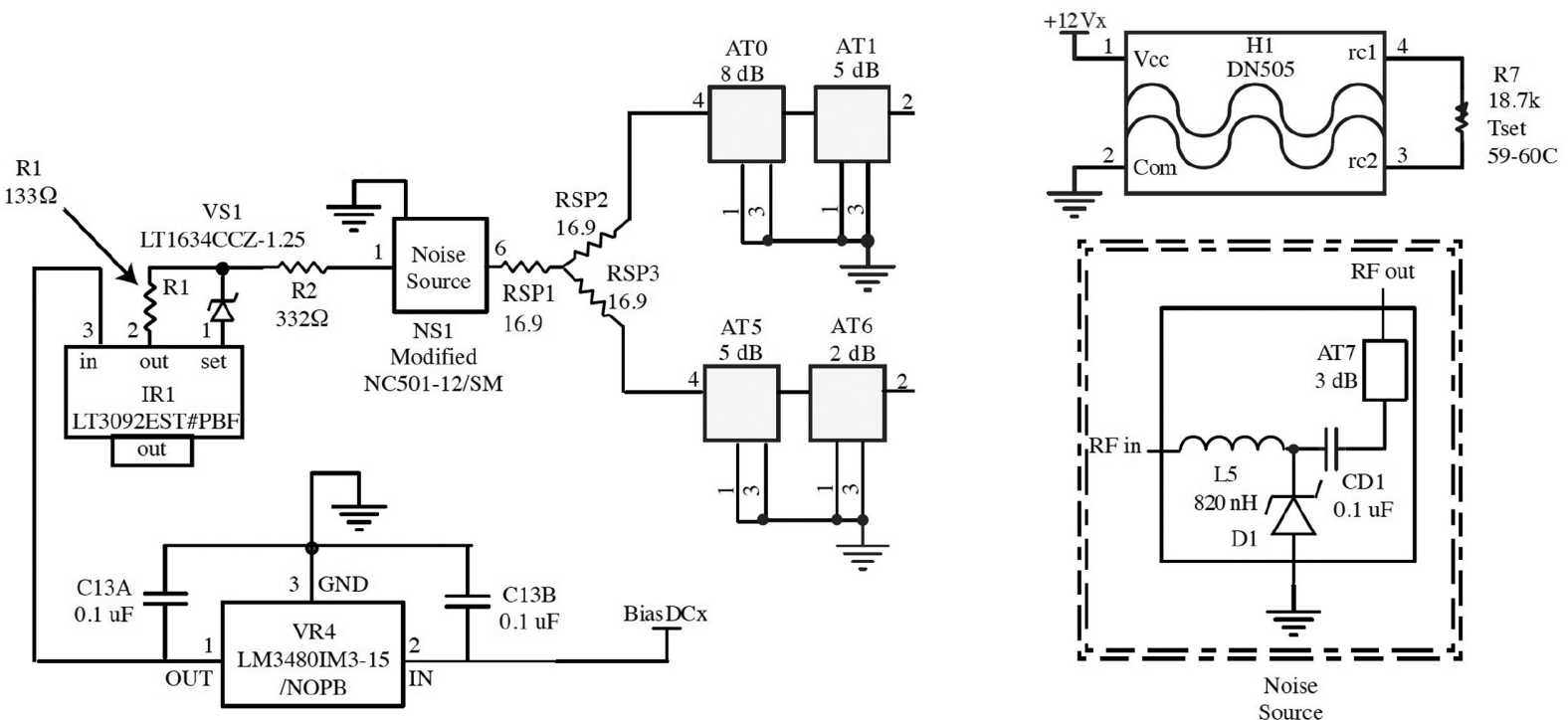

Figure 8. Circuit schematic for the calibration subcircuit. This circuit is housed in a Delrin shell; the DN505 heater is placed on top of the modified NC501 package. 


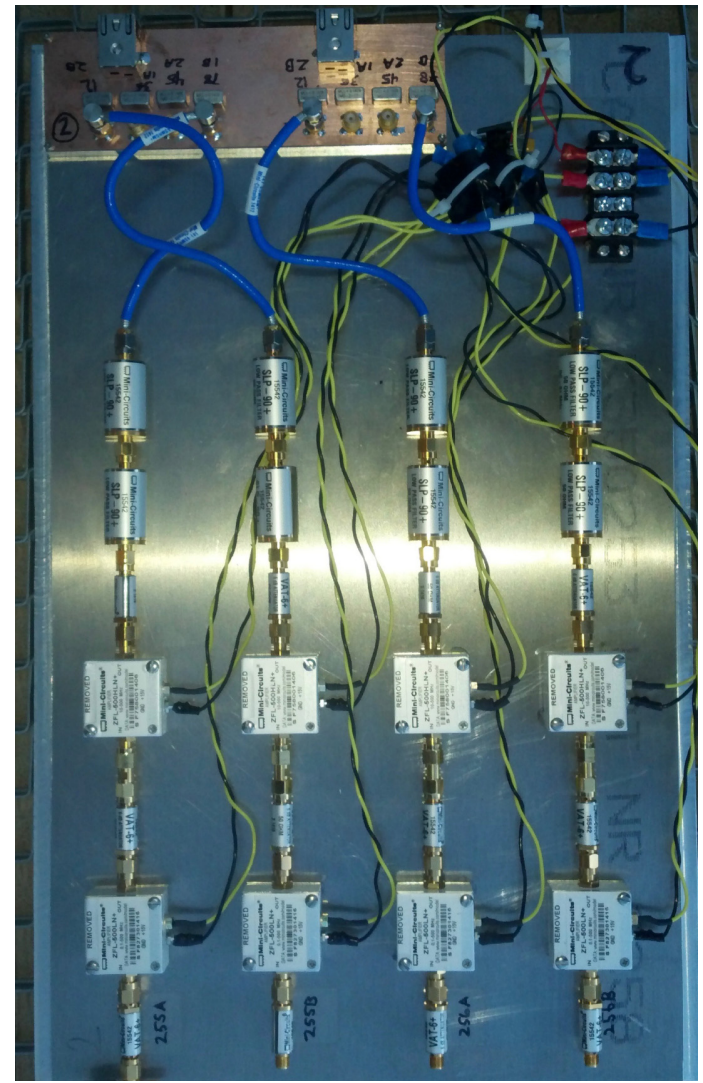

Figure 9. Four of 10 signal paths in the LEDA connectorized back-end electronics (CRX), which was adopted during an upgrade cycle in place of closely packed and unshielded LWA RF circuitry to reduce pickup of internally generated RFI and reflections.

ARJ45 was substituted for LWA-standard RJ45 CAT5e during an upgrade cycle, motivated by superior near-end crosstalk. Although the digitizer cards were not upgraded, VNA S21 bench measurements demonstrated crosstalk of $<-60 \mathrm{~dB}$ for a CAT7A signal path including a passthrough and single CAT5e plug and jack combination at one end. With this, residual crosstalk in the signal path is dominated by the analog-to-digital (ADC) card. Bench measurements have demonstrated reduction to between -30 and $-40 \mathrm{~dB}$ across the science band when only two of four conductor pairs are used on each cable, corresponding to CAT5e 8P8C connector pins 1 and 2 , and 7 and 8 which are physically the most widely separated.

\section{CALIBRATION EQUATIONS}

The output power of a radiometer system is given by

$P_{\text {out }}=G k_{\mathrm{B}} \Delta v\left(T_{\mathrm{ant}}+T_{\mathrm{rx}}\right)$

where $G$ is the total gain of the radiometer's analogue systems (amplifiers, filters, etc.), $k_{\mathrm{B}}$ is Boltzmann's constant, and $T_{\mathrm{rx}}$ and $T_{\mathrm{ant}}$ are the noise-equivalent temperatures of the receiver and antenna, respectively. At frequencies corresponding to the $\mathrm{CD}$ trough, radiometer systems are generally sky-noise dominated; that is, $T_{\text {ant }}$ $\gg T_{\mathrm{rx}}$. Equation (5) is the fundamental measurement made by a radiometer.

Antenna temperature is given by the average of the actual sky brightness $T_{\text {sky }}(\theta, \varphi)$ as seen from the antenna's location, weighted by the antenna's gain pattern $B(\theta, \varphi)$ :

$T_{\text {ant }}(v)=\frac{\int \mathrm{d} \Omega B(\theta, \phi, v) T_{\text {sky }}(\theta, \phi, v)}{\int \mathrm{d} \Omega B(\theta, \phi, v)}$.

If one separates the sky temperature $T_{\text {sky }}$ into a 'foreground' component, $T_{\text {fg }}$, and the cosmological, $T_{\text {cosm }}$, term consisting of the skyaveraged $21-\mathrm{cm}$ emission from equation (1) and the background $\mathrm{CMB}$ radiation, then

$T_{\text {sky }}(\theta, \phi, v)=T_{\text {fg }}(\theta, \phi, v)+T_{\text {cosm }}(v)$,

such that equation (6) becomes

$T_{\text {ant }}(v)=\frac{\int \mathrm{d} \Omega B(\theta, \phi, v) T_{\mathrm{fg}}(\theta, \phi, \nu)}{\int \mathrm{d} \Omega B(\theta, \phi, \nu)}+T_{\operatorname{cosm}}(\nu)$.

In practice, impedance mismatch between the antenna and receiver must also be taken into consideration, as must noise waves generated by the receiver's first-stage amplifier. These factors are detailed further in Section 5.

The LEDA radiometer employs a three-state switching calibration technique (Rogers \& Bowman 2012), where the receiver cycles between the sky and two calibration references (a 'hot' and 'cold' state). Three-state switching allows the removal of variations in system gain $G=G(v, t)$ and receiver temperature $T_{\mathrm{rx}}=T_{\mathrm{rx}}(\nu, t)$, and allows for a temperature scale to be imposed on the data.

The LEDA outrigger antennas switch between the sky and two calibration reference paths. A noise diode in series with attenuators is used to provide a reference with an equivalent noise temperature of $T_{\text {hot }}$ and $T_{\text {cold }}$. The power measured by the radiometer in each state (equation 5 ) is given by

$P_{\mathrm{ant}}=G k_{\mathrm{B}} \Delta v\left(T_{\mathrm{ant}}+T_{\mathrm{rx}}\right)$

$P_{\text {hot }}=G k_{\mathrm{B}} \Delta v\left(T_{\mathrm{hot}}+T_{\mathrm{rx}}\right)$

$P_{\text {cold }}=G k_{\mathrm{B}} \Delta v\left(T_{\text {cold }}+T_{\mathrm{rx}}\right)$,

where $P_{\text {ant }}, P_{\text {hot }}$, and $P_{\text {cold }}$ are powers measured in antenna, hot reference, and cold reference states; $T_{\text {ant }}, T_{\text {hot }}$, and $T_{\text {cold }}$ are antenna, hot, and cold reference noise-equivalent temperatures. The threestate switch calibrated temperature $T_{\text {ant }}$ may then be recovered via

$T_{\text {ant }}=\left(T_{\text {hot }}-T_{\text {cold }}\right) \frac{P_{\text {ant }}-P_{\text {cold }}}{P_{\text {hot }}-P_{\text {cold }}}+T_{\text {cold }}$.

Example spectra for the three states, is shown in Fig. 10. As presented in Rogers \& Bowman (2012), the true antenna temperature $T_{\text {cant }}$ is related to the three-state calibrated $T_{\text {ant }}$ by

$T_{\text {cant }}=T_{\text {ant }}\left(1-|\Gamma|^{2}\right)$,

where $\Gamma$ is the reflection coefficient: a measure of impedance mismatch between the receiver and the antenna.

However, equation (13) is not strictly accurate for two reasons. First, one must take care to use an appropriate definition for power gain $G$, for which there are several (see e.g. Pozar 2005). Here, we are interested in the power delivered to the load from a given source, for which the transducer power gain should be used. As shown in Pozar (2005), for a given amplifier with $S_{12}$ (reverse isolation, see Section 6.2 for more details) negligibly small, when connected to a source with reflection coefficient $\Gamma_{\mathrm{S}}$ and a load with reflection coefficient $\Gamma_{\mathrm{L}}$, the transducer power gain is given by:

$G_{\mathrm{T}}=\frac{\left|S_{21}\right|^{2}\left(1-\left|\Gamma_{\mathrm{S}}\right|^{2}\right)\left(1-\left|\Gamma_{\mathrm{L}}\right|^{2}\right)}{\left|1-S_{11} \Gamma_{\mathrm{S}}\right|^{2}\left|1-S_{22} \Gamma_{\mathrm{L}}\right|^{2}}$, 

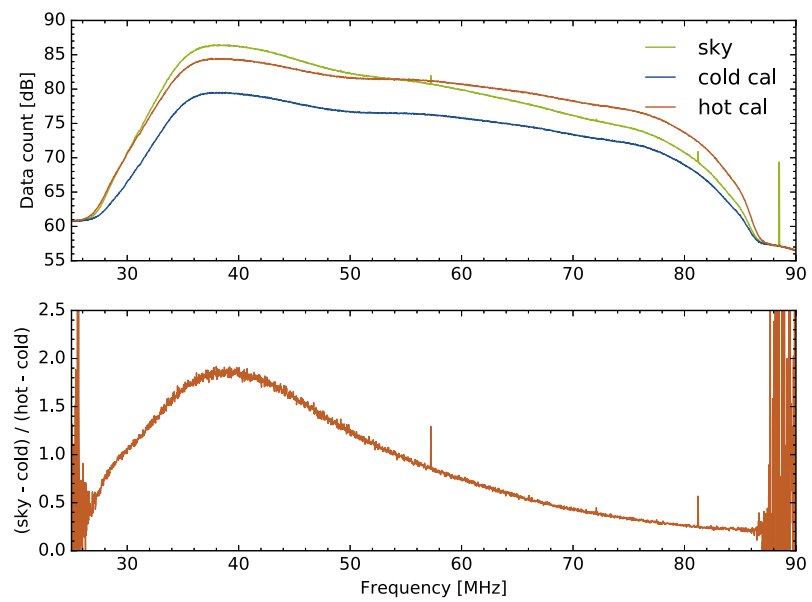

Figure 10. Top: typical bandpasses of antenna 252A in sky and calibration states, i.e. $P_{\text {ant }}, P_{\text {cold }}$, and $P_{\text {hot }}$. Bottom: the ratio $\left(P_{\text {ant }}-P_{\text {cold }}\right) /\left(P_{\text {hot }}-P_{\text {cold }}\right)$ of data in top panel.

where $S_{21}$ is a parameter equivalent to forward gain, and $S_{11}$ and $S_{22}$ are reflection coefficients. Note that in the ideal case, $\Gamma_{\mathrm{L}}=\Gamma_{\mathrm{S}}=0$ and equation (14) yields $G_{\mathrm{T}}=\left|S_{21}\right|^{2}$.

Secondly, the noise temperature of the receiver, $T_{\mathrm{rx}}$, also depends upon the source. For an amplifier with optimal noise figure $F_{\text {opt }}$, the noise figure $F$ for a given $\Gamma_{\mathrm{S}}$ is given by

$F=F_{\mathrm{opt}}+\frac{4 R_{\mathrm{N}}}{Z_{0}} \frac{\left|\Gamma_{\mathrm{S}}-\Gamma_{\mathrm{opt}}\right|^{2}}{\left(1-\left|\Gamma_{\mathrm{S}}\right|^{2}\right)\left|1+\Gamma_{\mathrm{opt}}\right|^{2}}$

where $R_{\mathrm{N}}$ is the equivalent noise resistance of the amplifier, $Z_{0}$ is the characteristic impedance, and $\Gamma_{\mathrm{opt}}$ is the amplifier's reflection coefficient at which its noise figure is the lowest. The receiver temperature - defined as $T_{\mathrm{rx}}=T_{0}(F-1)$ with $T_{0}=290 \mathrm{~K}-$ is thus dependent upon the source. An alternative approach to modelling noise within an analogue system is to use noise correlation matrices (King 2010; King et al. 2015), or 'noise wave' analysis (Meys 1978); the EDGES formalism uses the latter approach.

The magnitude of the inaccuracy of equation (12) due to neglect of equations (14) and (15) is primarily dependent upon $\Gamma_{\mathrm{S}}$. The formalism of Rogers \& Bowman (2012) (and Monsalve et al. 2017) is therefore only accurate in the case that the diode and load states are well matched to the receiver; in both the LEDA and EDGES instruments, $\Gamma_{\mathrm{S}}$ is lower than $-30 \mathrm{~dB}$ for reference states, so this requirement is satisfied.

For the calibration detailed here, we follow the formalism of Rogers \& Bowman (2012), but nonetheless highlight that improvement of the formalism is an area deserving future examination. Following this formalism, $\Gamma$ in equation (13), is calculated from reflection coefficients of the antenna and receiver, $\Gamma_{\text {ant }}$ and $\Gamma_{\mathrm{rx}}$ respectively (Fig. 5), measured with a vector network analyser (VNA), and

$$
\begin{aligned}
T_{\text {cant }}= & T_{\text {sky }} H_{\text {ant }}|F|^{2} H_{\mathrm{rx}}^{-1} \\
& +T_{\mathrm{u}}\left|\Gamma_{\mathrm{ant}}\right|^{2}|F|^{2} H_{\mathrm{rx}}^{-1} \\
& +\left(T_{\mathrm{c}} \cos (\psi)+T_{\mathrm{s}} \sin (\psi)\right)\left|\Gamma_{\mathrm{ant}}\right||F| H_{\mathrm{rx}}^{-1} .
\end{aligned}
$$

The terms of equation (16) are:

(i) $\Gamma_{\text {ant }}$ is the reflection coefficient of the antenna, as measured at the output of the balun. (ii) $\Gamma_{\mathrm{rx}}$ is the reflection coefficient of the receiver. The first component in the receiver is an LNA, which will be a main cause of reflections between the antenna and the receiver.

(iii) $H_{\mathrm{ant}}=1-\left|\Gamma_{\mathrm{ant}}\right|^{2}$ and $H_{\mathrm{rx}}=1-\left|\Gamma_{\mathrm{rx}}\right|^{2}$ are gain terms arising due to antenna / receiver mismatch.

(iv) $F=\left(1-\left|\Gamma_{\mathrm{rx}}\right|^{2}\right)^{1 / 2}\left(1-\Gamma_{\mathrm{ant}} \Gamma_{\mathrm{rx}}\right)^{-1}$ is another complex gain factor encompassing receiver and antenna mismatch.

(v) $T_{\mathrm{u}}$ is the uncorrelated 'noise wave' power, emitted from the LNA and reflected back by the antenna (see Meys 1978, for noise wave formulation).

(vi) $T_{\mathrm{c}} \cos (\psi)$ and $T_{\mathrm{s}} \sin (\psi)$ are correlated noise waves that depend on the amplitude and phase of the antenna reflection, where $\psi$ is the phase of the noise wave reflected from the antenna.

From equation (16), one may solve for the true sky temperature $T_{\text {sky }}$. If the antenna is not lossless, a further correction must be applied:

$T_{\text {csky }}=\left(T_{\text {sky }}-T_{\text {amb }}(1-L)\right) / L$,

where $L=10^{-l / 10}$ for a loss $l$ in $\mathrm{dB}$, and $T_{\mathrm{amb}}$ is the ambient temperature of the antenna.

\subsection{Thermal uncertainties}

The LEDA receiver uses two reference diode states, in contrast to the load and diode approach used in EDGES. Here, we show that the dual diode approach optimizes measurement $\mathrm{S} / \mathrm{N}$.

An estimate of thermal noise present in the three-state switched spectrum may be found by propagating the uncertainties of equation (12):

$\mathrm{d} T_{\text {ant }}^{2}=\left(\frac{\partial T_{\text {ant }}}{\partial P_{\text {ant }}}\right)^{2} \mathrm{~d} P_{\text {ant }}^{2}+\left(\frac{\partial T_{\text {ant }}}{\partial P_{\text {cold }}}\right)^{2} \mathrm{~d} P_{\text {cold }}^{2}+\left(\frac{\partial T_{\text {ant }}}{\partial P_{\text {hot }}}\right)^{2} \mathrm{~d} P_{\text {hot }}^{2}$.

where the uncertainty of measurement in each state is given by the radiometer equation. This yields

$\mathrm{d} T_{\text {ant }}=A \sqrt{\mathrm{d} P_{\text {ant }}^{2}+B\left(\mathrm{~d} P_{\text {hot }}\right)^{2}+C\left(\mathrm{~d} P_{\text {cold }}\right)^{2}}$

$A=\frac{T_{\text {hot }}-T_{\text {cold }}}{P_{\text {hot }}-P_{\text {cold }}}$

$B=\left(\frac{P_{\text {ant }}-P_{\text {cold }}}{P_{\text {hot }}-P_{\text {cold }}}\right)^{2}$

$C=\left(\frac{P_{\text {ant }}-P_{\text {hot }}}{P_{\text {hot }}-P_{\text {cold }}}\right)^{2}$

It follows that to optimize measurement $\mathrm{S} / \mathrm{N}$, the two references should be as high power as possible, while maintaining a large difference in power between them. In tension with this, the finite dynamic range of the ADC motivates diode temperatures comparable to the sky brightness. Fig. 11 compares the measurement uncertainty as a function of observation time for a dual diode system with fiducial values $T_{\text {hot }}=6500 \mathrm{~K}$ and $T_{\text {cold }}=1000 \mathrm{~K}$, against a system with $T_{\text {hot }}=450 \mathrm{~K}$ and $T_{\text {cold }}=300 \mathrm{~K}$.

\section{INSTRUMENT CHARACTERIZATION}

Of particular importance to calibration is a sound understanding of the characteristics of the FE. In this section, we present detailed measurements of the characteristics of the FE and antenna for a single polarization (antenna 252A). None the less, all FE boards undergo the same characterization process; comparison between antennas is presented in following sections. 


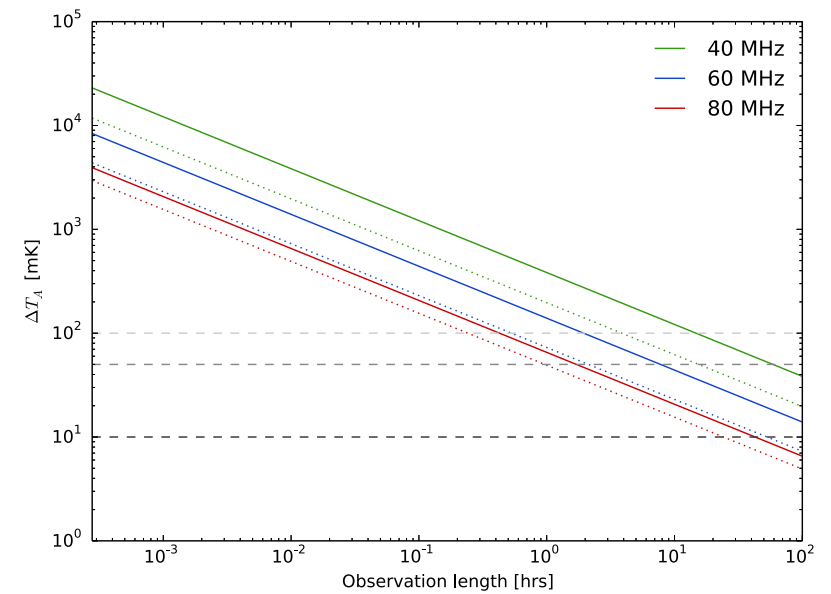

Figure 11. Expected improvement of measurement residuals for a system using dual noise diode references, based on use of equation (18). The dotted lines represent a system with $T_{\text {hot }}=6500 \mathrm{~K}$ and $T_{\text {cold }}=1000 \mathrm{~K}$; solid lines represent a system with $T_{\text {hot }}=450 \mathrm{~K}$ and $T_{\text {cold }}=300 \mathrm{~K}$.

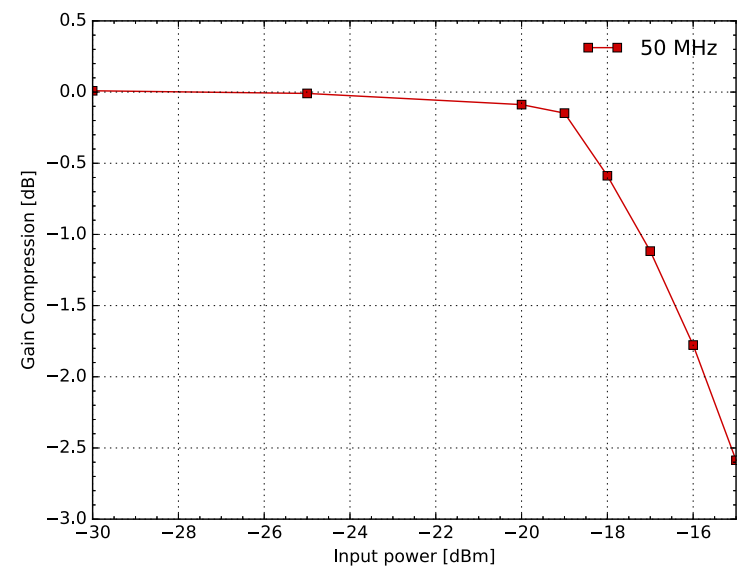

Figure 12. Gain compression curve for the LEDA receiver at $50 \mathrm{MHz} .1 \mathrm{~dB}$ compression occurs at an input power of $-17.2 \mathrm{dBm}$.

\subsection{Gain linearity}

To ensure the amplifiers on the FE are operating in a nominal regime, we tested the gain linearity of the FE using an Agilent E4424B signal generator and Agilent N9000A spectrum analyser. The signal generator was used to produce a $50 \mathrm{MHz}$ tone with amplitudes covering -100 to $-15 \mathrm{dBm}$. We found the $1 \mathrm{~dB}$ compression point occurs at an input power of $-17.2 \mathrm{dBm}$ (Fig. 12). Note that the expected power from the antenna is well under this $1 \mathrm{~dB}$ compression point: for a $10000 \mathrm{~K}$ sky over $100 \mathrm{MHz}$ (an overestimation), one would expect $-78.6 \mathrm{dBm}$ input power to the FE.

The first harmonic of the $50 \mathrm{MHz}$ tone was not apparent on the spectrum analyser until an input power of $-40 \mathrm{dBm}$ (far above expected input power), when it appeared above the spectrometer's noise floor with an output power of $-72.8 \mathrm{dBm}$. From our data, we extrapolate the IP2 intercept to be $\sim 37 \mathrm{dBm}$.

\subsection{Scattering parameters}

The reflection and transmission characteristics of the LEDA FE and LWA antenna were measured using an Anritsu MS2034B VNA. Between the MS147 test ports and the SMA output, the LEDA FE

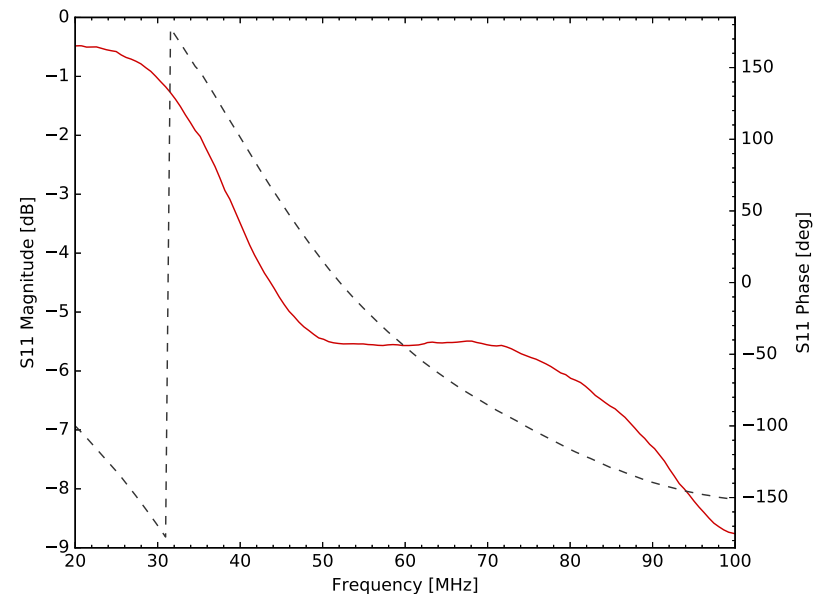

Figure 13. Magnitude (red) and phase (black dashed) of reflection coefficient $\Gamma_{\text {ant }}$ for antenna 252A. These data were taken in 2016 January.

can be treated as a two-port network. This allows us to measure its scattering parameters (S-parameters) relating incident and reflected voltage waves.

\subsubsection{Antenna + balun}

A VNA measurement of the antenna cannot be made without the use of a balun; the characteristics of the balun must therefore be known for one to de-embed its effect. The MS147 connector directly after the FE balun allows the reflection coefficient $\Gamma_{\text {ant }}$ to be measured, see Fig. 5.

Given the far-field distance of the antennas is many hundreds of metres, and that the surrounding environment affects the beam characteristics, data were necessarily taken in situ at OVRO. Care was taken to ensure that during measurement, all equipment was placed low to the ground as far away as possible. The VNA was placed on the ground, at a distance of $20 \mathrm{~m}$ away from the antenna, orthogonal to the antenna blade pair under test. Low-loss coaxial cable was laid from the VNA, across the ground, and up the antenna's central mast to the FE MS147 connector that connects to the balun.

The magnitude and phase of $\Gamma_{\text {ant }}$ for antenna 252A is shown in Fig. 13. The magnitude and phase of $\Gamma_{\text {ant }}$ are seen to vary smoothly as a function of frequency, varying between -4 to $-6 \mathrm{~dB}$ over the 40-85 MHz band.

\subsubsection{Front-end receiver}

S-parameter measurements of the FE were taken by connecting the VNA to the MS147 connector directly preceding the switch. This port is labelled $\Gamma_{\mathrm{sw} 0}$ in Fig. 5; we will refer to this as $\Gamma_{\mathrm{rx}}$, as it is the main VNA measurement presented for the receiver board. The board was characterized over $10-100 \mathrm{MHz}$, using a low VNA port power $(-25 \mathrm{dBm})$ such that the FE was operating in a linear gain regime. So that the FE could be measured as a single device under test (DUT), the board was powered via the regulator daughter board in lieu of using an external bias tee.

The S-parameters for the FE are shown in Fig. 14. The overall gain $\left(S_{21}\right)$ is $32.1 \pm 0.2 \mathrm{~dB}$ over $30-80 \mathrm{MHz}$. The 3 and $10 \mathrm{~dB}$ roll-off points occur at $(27.7,85.5) \mathrm{MHz}$ and $(26.0,88.7) \mathrm{MHz}$, respectively; primarily due to the bandpass filter. The $S_{11}$ (i.e. $\Gamma_{\text {ant }}$ ), is better than $-30 \mathrm{~dB}$ across the LEDA science band of $40-85 \mathrm{MHz}$, 

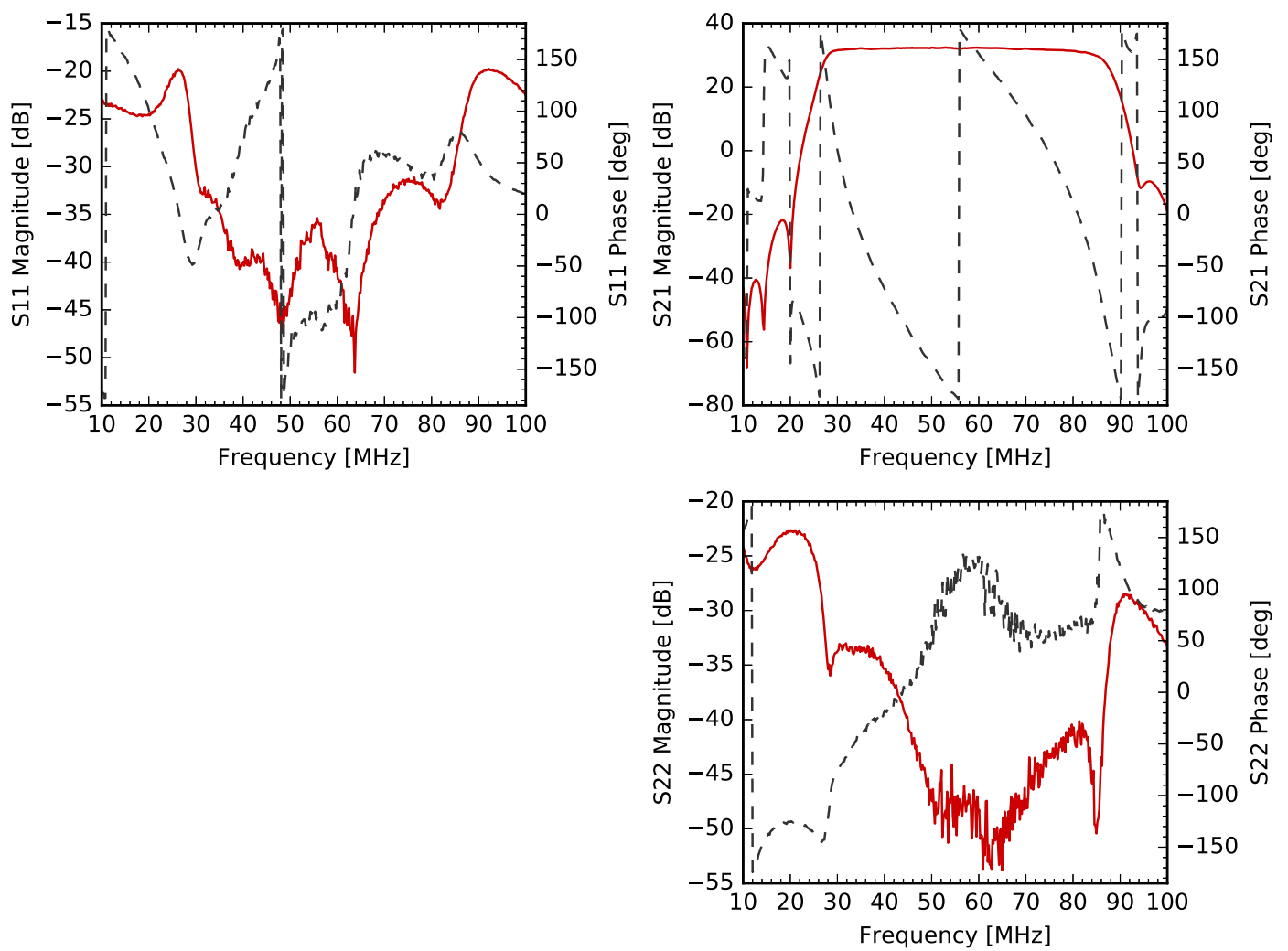

Figure 14. Magnitude (red) and phase (dashed black) of scattering parameters for LEDA FE installed on antenna 252A. The $S_{12}$ parameter is below the measurement ability of the VNA (below $-60 \mathrm{~dB}$ ) so is not shown.

with $S_{11}$ increasing outside of the passband. Similarly, the $S_{22}$ is also better than $-30 \mathrm{~dB}$ across the LEDA science band.

\subsubsection{Calibration parameters}

From the VNA measurements of the FE and antenna, we are able to form the calibration parameters $H_{\mathrm{ant}}, H_{\mathrm{rx}}$, and $|F|^{2}$ (Fig. 15). In the top panels of Fig. 15, the VNA measurements are shown in red, and smoothly varying fitted models are shown in black; the bottom panel shows the residual between the VNA data and the fit. We have fit $H_{\mathrm{rx}}$ with an 11-term polynomial, $|F|^{2}$ with a 21 -term Fourier series, and $H_{\text {ant }}$ with a combined five-term polynomial and 21-term Fourier series. As such, $H_{\text {ant }}$ has the largest effect upon calibration; the LNA's low reflection coefficient $\Gamma_{\mathrm{rx}}$ means that $H_{\mathrm{rx}}$ has only a small $(<0.1$ per cent $)$ effect upon the overall calibration.

\subsection{Receiver temperature}

We determined the receiver temperature $T_{\mathrm{rx}}$ using the Y-factor method (Pozar 2005). For an accurate measurement, noise contribution and any loss from the cables and connectors between the reference source and DUT must be included in $T_{\text {hot }}$ and $T_{\text {cold }}$. Further, precise measurement requires $Y \gg 1$, meaning the hot and cold references should be as different as possible.

To measure the receiver temperature of the LEDA receiver boards (DUT), we applied the Y-factor method using a calibrated HP 346C noise source as a reference. Hot and cold references states were created by inserting a 10 and $6 \mathrm{~dB}$ pad between the $346 \mathrm{C}$ and the DUT; the resulting noise temperature was computed using

$T_{\text {cal }}=\left(1-L_{\text {att }}\right) T_{346 \mathrm{C}}+290 L_{\text {att }}$ where $L_{\text {att }}$ is the combined loss of the attenuator and coaxial cable, as measured using a VNA, and $T_{346 \mathrm{C}}$ is the manufacturer specified noise temperature of the HP $346 \mathrm{C}$ source.

We measured the $T_{\mathrm{rx}}$ of all FE boards in the laboratory immediately prior to installation, using an Agilent 9000A spectrum analyser; the HP346C reference source was connected at the MS147 test port between the balun and the switch. We find the receiver temperatures to be in good agreement with Table 2 within 30$80 \mathrm{MHz}$, increasing rapidly outside the receiver passband (Fig. 16). For data analysis, we fit a line to the measured $T_{\mathrm{rx}}$ between 40 and $80 \mathrm{MHz}$.

\subsubsection{Noise diode temperatures}

Once the receiver temperature is known, the temperature of the hot and cold references can be calculated with reference to the external HP346C. Fig. 17 shows the equivalent noise temperature of the hot (red) and cold (blue) noise diode reference states. A linear model is fitted to both states (black), which is used in subsequent calibration.

\subsection{LNA noise wave analysis}

As discussed in Section 5, calibration requires that the noise waves emitted by the receiver are accounted for (equation 12). Characterization of the receiver's emitted noise wave (see Meys 1978) requires multiple measurements of output power with varying impedances at the receiver's input. A convenient method for characterization of the noise wave is by applying equation (12) to a system where the antenna is replaced by an open (or shorted) coaxial cable; the wrapping of phase $\varphi$ as a function of frequency over a cable of 

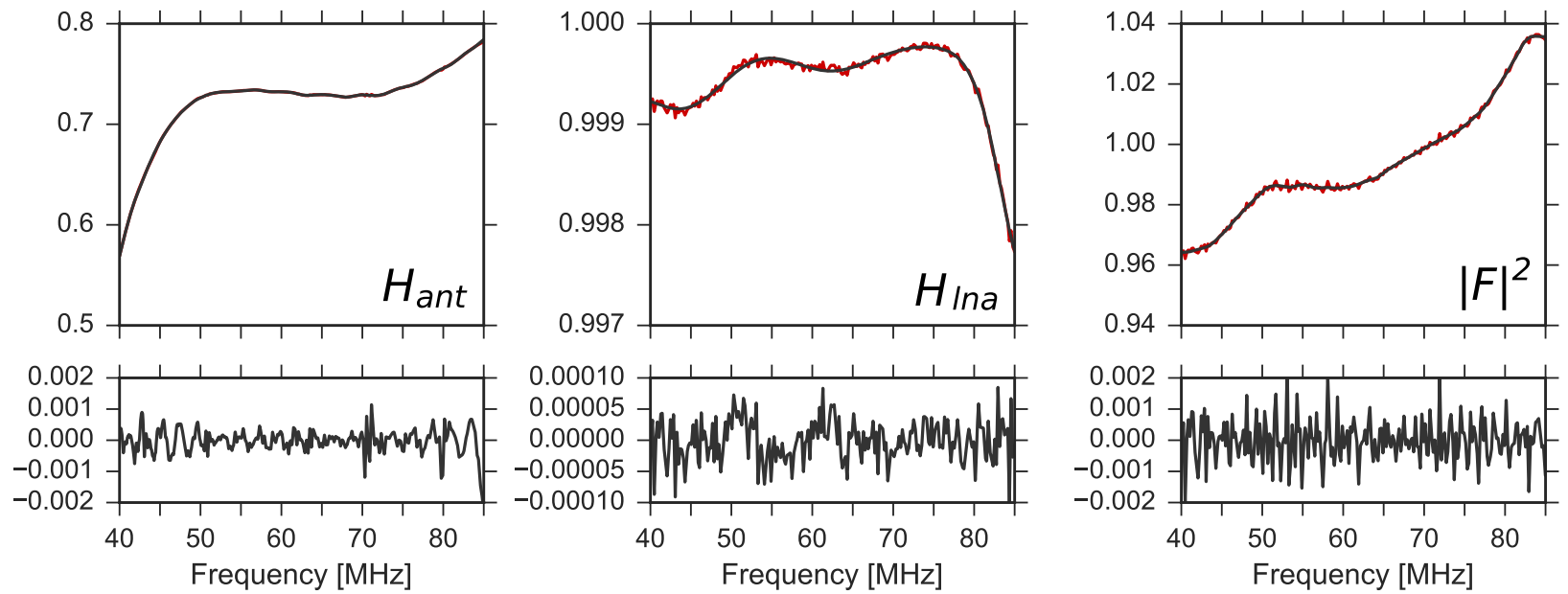

Figure 15. Calibration parameters $H_{\mathrm{ant}}, H_{\mathrm{rx}}$, and $|F|^{2}$ (left to right). In the top panels, VNA measurements are shown in red, with a model fitted in black. The bottom panel shows the residual between the VNA measurements and the model.

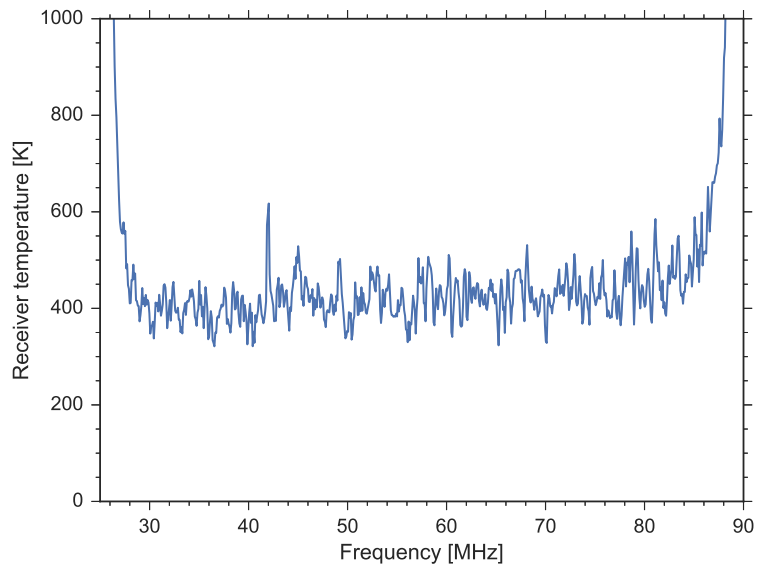

Figure 16. LEDA FE receiver temperature for antenna 252A.

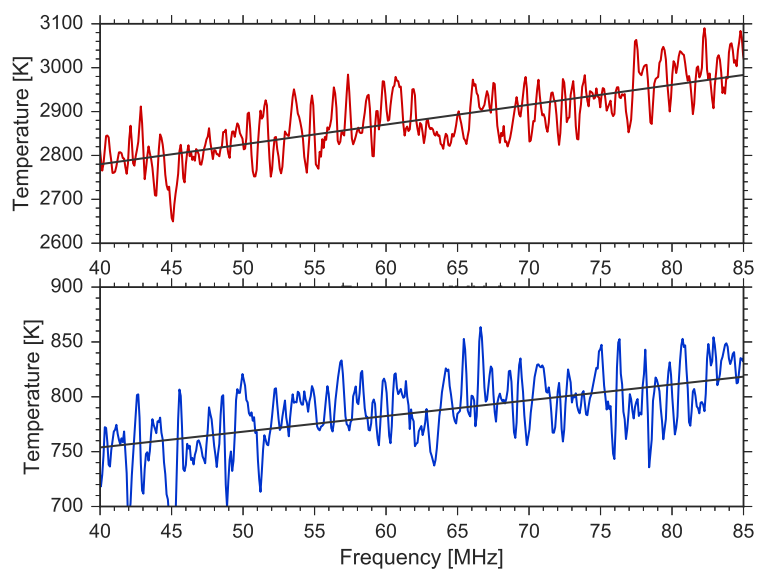

Figure 17. LEDA FE noise diode temperatures for antenna 252A.

suitable length allows deduction of the phase of the emitted noise wave. The process we employed was as follows:

(i) The reflection coefficient $\Gamma_{\text {coax }}$ of an open coaxial cable at room temperature was measured using a VNA, along with $\Gamma_{\mathrm{rx}}$.
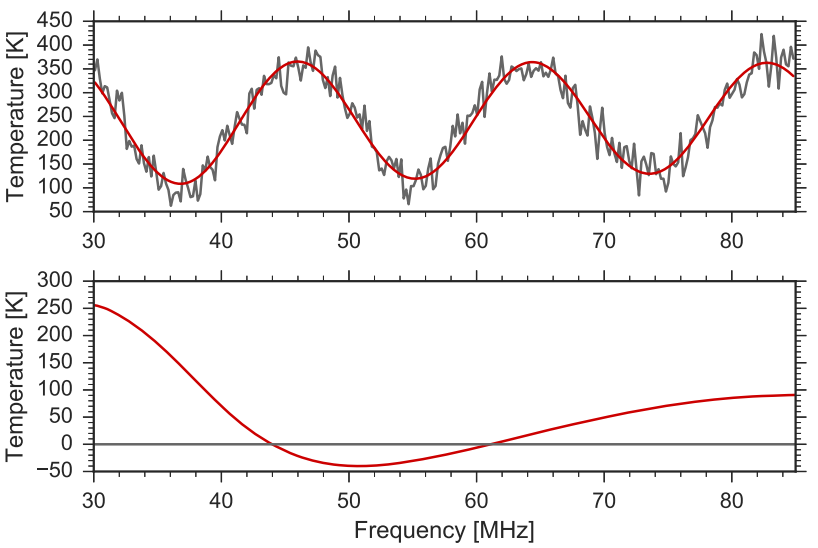

Figure 18. Top: spectra of coaxial cable used to characterize FE receiver noise wave, with measured spectra in grey and fitted model for scalar values of $T_{\mathrm{u}}, T_{\mathrm{c}}, T_{\mathrm{s}}$, and $T_{0}$ in red. Bottom: computed contribution of FE noise waves for antenna $252 \mathrm{~A}$.

(ii) The coaxial cable was connected to the FE at the MS147 test port, and power spectra for $P_{\text {coax }}, P_{\text {hot }}$, and $P_{\text {cold }}$ were measured using a spectrum analyser.

(iii) A three-state calibrated spectrum was computed via application of equation (12) (top panel of Fig. 18).

(iv) Replacing the terms $\Gamma_{\text {ant }}$ and $H_{\text {ant }}$ with $\Gamma_{\text {coax }}$ and $H_{\text {coax }}$, we applied least-squares fitting to estimate scalar values $T_{\mathrm{c}}, T_{\mathrm{s}}, T_{\mathrm{u}}$, and $\psi$.

For the FE corresponding to antenna 252A, we measure $T_{\mathrm{U}}=194.67, T_{\mathrm{c}}=-174.39$, and $T_{\mathrm{s}}=-1.14$. The overall magnitude of the noise wave from the receiver, when connected to antenna $252 \mathrm{~A}$, is shown in the bottom panel of Fig. 18 and is a level of a few per cent of the sky temperature.

\subsection{Noise diode thermal stability}

The characteristics of many active RF devices, including noise diodes, are dependent upon ambient temperature. We used a thermal control chamber (Test Equity 1000) to characterize the effect of ambient temperature upon the output power of the FE noise diode. The 


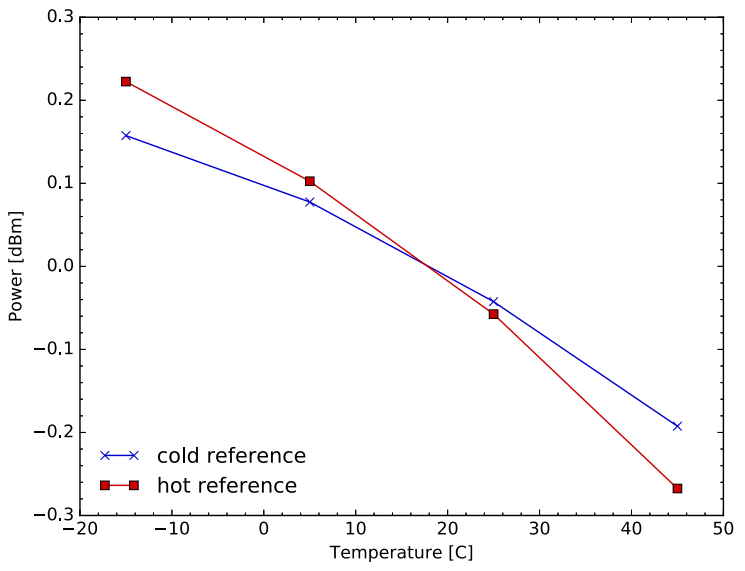

Figure 19. Change in noise diode output power as a function of temperature, as measured over hot (red) and cold (blue) reference paths.

chamber allows control of the ambient temperature from $-15^{\circ} \mathrm{C}$ to $45^{\circ} \mathrm{C}$, with $\pm 0.1^{\circ} \mathrm{C}$ precision.

The FE was placed in the chamber inside an RF-shielded box; a coaxial cable connected the FE output to an HP436A power meter via a bias tee that supplied DC voltage to the FE. The power meter also outputs a $0 \mathrm{dBm} 50 \mathrm{MHz}$ reference tone; this was connected to the FE input MS147 port, with $60 \mathrm{~dB}$ of attenuation was added at the reference output. We waited 20 min between temperature changes, to allow the FE and RF-tight box to equilibrate.

We found a temperature coefficient of $-0.00815 \mathrm{~dB} \mathrm{~K}^{-1}$ for the hot reference path, and $-0.00585 \mathrm{~dB} \mathrm{~K}^{-1}$ for the cold path (Fig. 19). The fractional stability of the two paths is 0.00874 per cent; as such, temperature dependence of the noise diode is not expected to be a significant source of error.

\subsection{Allan deviation}

The Allan variation, $\sigma_{\mathrm{y}}^{2}(\tau)$, and the Allan deviation, $\sigma_{\mathrm{y}}(\tau)=$ $\sqrt{\sigma_{\mathrm{y}}^{2}(\tau)}$, are common measures of stability over time. Allan deviation may be used to differentiate between different types of noise within a system. Notably, for random Gaussian noise, such as that in a radiometer, the Allan deviation will decrease as $\tau^{-1 / 2}$.

To characterize the stability of the LEDA radiometer system in the field, we took spectrometer data overnight with a $50 \Omega$ load connected to the FE MS147 input port. Data were calibrated using the three-state switching method outlined above. We used the ALLANTooLs package (Wallin et al. 2018) to compute the Allan deviation for the calibrated overnight data (Fig. 20), finding a maximum integration time of $\tau=2000 \mathrm{~s}$, before other system instabilities become significant.

By equation (4), for $\Delta v=1 \mathrm{MHz}$ and sky temperatures of 5000, 3000 , and $1000 \mathrm{~K}$, the corresponding rms noise levels are 290, 174, and $58 \mathrm{mK}$, respectively. As such, the radiometer is stable enough to reach the level required for validation of the Bowman et al. (2018) result. Nevertheless, as will be discussed later, other systematics currently dominate the noise budget. We believe the main source of instability in this field test is the change in the load's ambient temperature overnight, and that the intrinsic stability is higher than that presented here; thermal isolation of the load will be required for future tests.

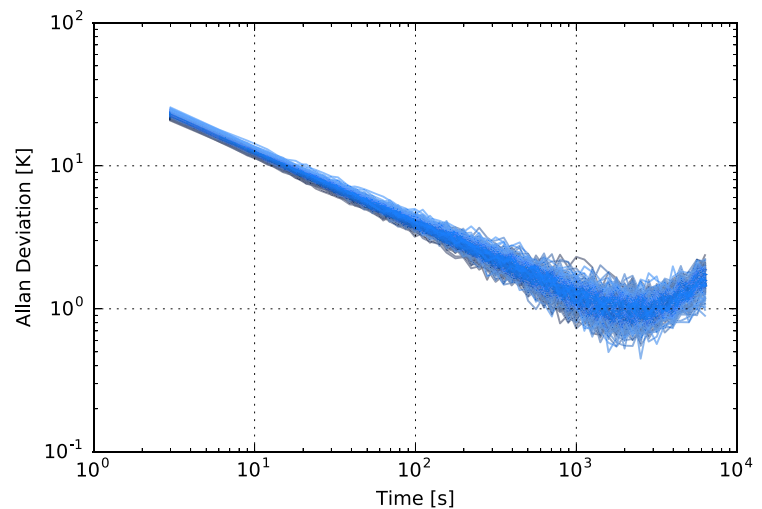

Figure 20. Allan deviation for the receiver system connected to a $50 \Omega$ load in place of the antenna. Every seventh spectrometer channel is plotted ( $\Delta \nu=24 \mathrm{kHz}$ ) between 30 and $80 \mathrm{MHz}$. Data were in the field, with the receiver board installed at the antenna.

\section{RESULTS}

Three LEDA FE boards, as described in Section 5, were deployed at OVRO-LWA in 2016 January, to antennas 252, 254 and 255 (Fig. 2). On 2016 January 27, on-sky data were recorded for 24 $\mathrm{h}$ using the LEDA digital spectrometer systems (Section 3.2). For these observations, we switched between the sky and the reference diode states every $5 \mathrm{~s}$. For reproducibility, these data, along with analysis scripts used to generate plots in this paper are available online. $^{2}$

During the 2016 January deployment, the 'B' polarization of board 252 was found to have poor characteristics, so have been excluded from analysis here. In this section, we first present detailed results from a single antenna, before comparing results across antennas in Section 7.5.

\subsection{Absolute calibration}

Data were calibrated using equation (16), following the measurement procedures outlined in Section 6. Fig. 21 shows the dynamic spectra for antenna 252A over the 24-h period on 2016 January 26, after RFI flagging (see Section 7.2). The corresponding antenna temperature spectrum at LST 11:00, is shown in the top panel of Fig. 22; and the bottom panel shows the change in system temperature over the $24-\mathrm{h}$ period at $60 \mathrm{MHz}$.

\subsection{RFI environment}

To identify and flag RFI, we apply the SUMTHRESHOLD algorithm (Offringa et al. 2010), which we have ported to a PYTHON package called DPFLGR. Dynamic spectra from antenna 252A post-flagging are shown in Fig. 21; and the flagged data fractions for day and night are shown in Fig. 23. The RFI environment is seen to be quieter at night, but nevertheless several bright narrow-band sources are omnipresent. We choose to completely flag channels or timesteps with high occupancy ( $>40$ per cent). The presence of increased RFI during the day, along with increased air traffic, onsite human activity, and potential solar flare events, and motivate primary LEDA observations to be conducted at night.

\footnotetext{
${ }^{2}$ http://github.com/telegraphic/leda_analysis_2016
} 


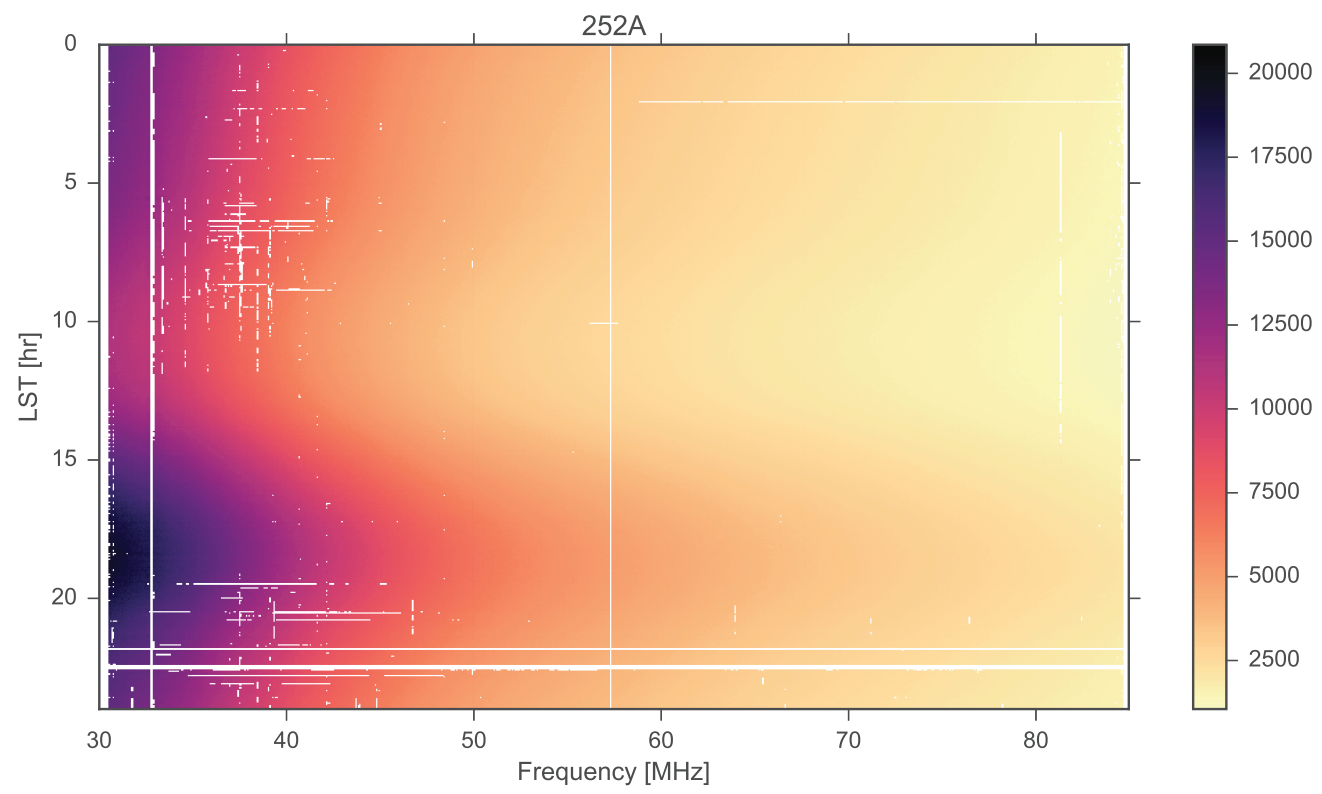

Figure 21. Dynamic spectra for antenna 252A on 2016 January 26 after RFI flagging. Flagged data are shown in white; and colour mapping is in Kelvin.
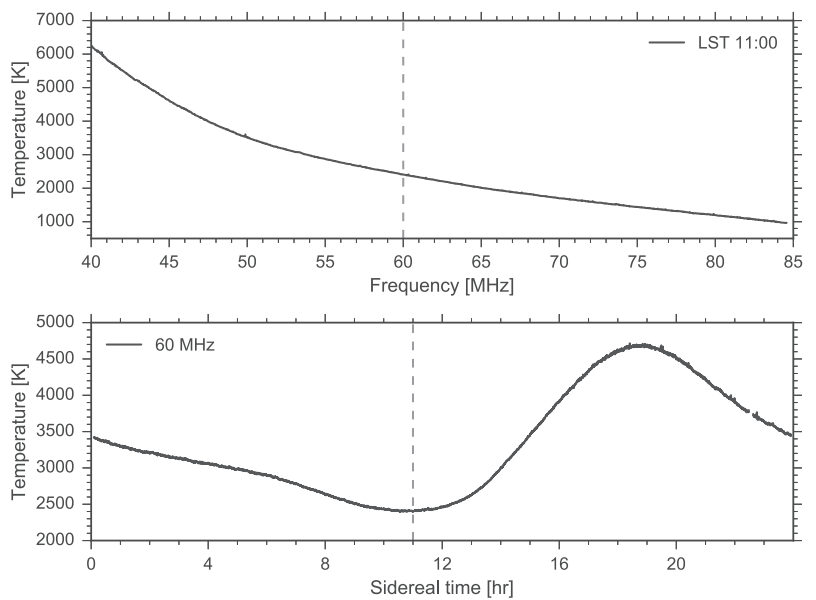

Figure 22. Top: measured antenna temperature for antenna 252A at LST 11:00, 2016 January 26. Bottom: measured antenna temperature at $60 \mathrm{MHz}$ over a 24 -h period.

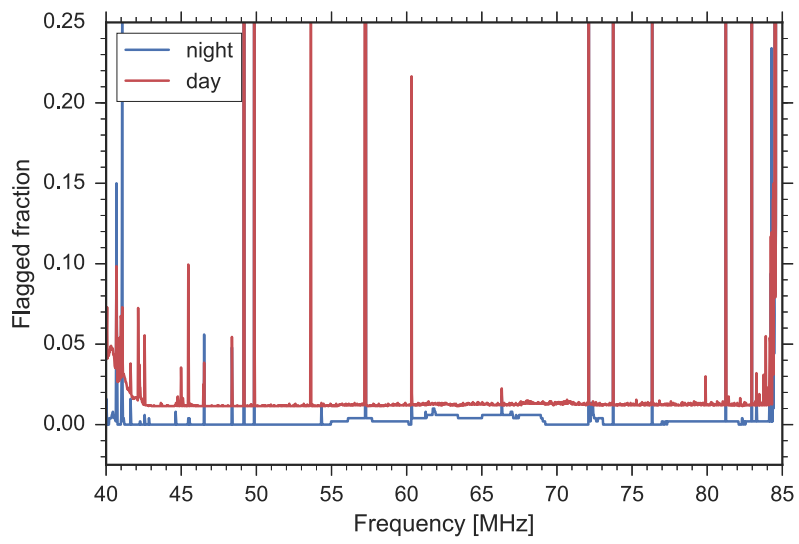

Figure 23. Fraction of data flagged by DPFLGR for day (red) and night (blue).

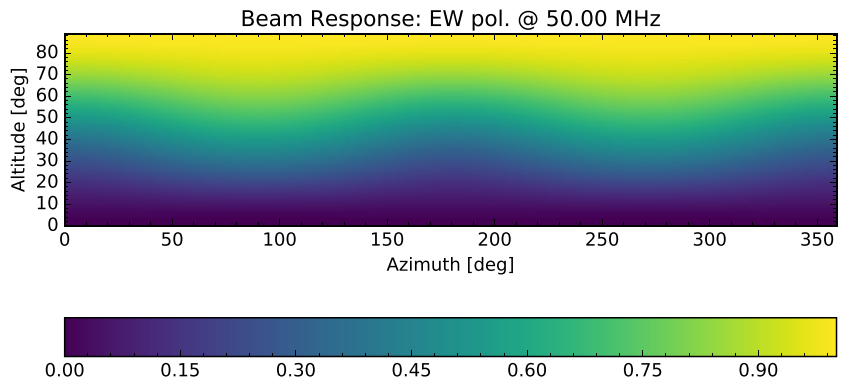

Figure 24. Empirical model of the LWA dipole antenna pattern at $50 \mathrm{MHz}$ for the east-west polarization (Dowell et al. 2017).

\subsection{Comparison to sky models}

To compare against our measurements, we simulated the expected antenna temperature spectra using a model of the LWA antenna with several sky models: the aforementioned GSM (GSM2008, de Oliveira-Costa et al. 2008), the 'updated' GSM released in 2016 (GSM2016, Zheng et al. 2017), and the Low-frequency Sky Model (LFSM, Dowell et al. 2017). For the antenna gain pattern, we used an empirical model (valid between 40 and $80 \mathrm{MHz}$ ) based on LWA1 data (Dowell et al. 2017); the response at $50 \mathrm{MHz}$ is shown in Fig. 24.

Simulated antenna temperature spectra for the three models are shown in Fig. 25, for an observer at OVRO, LST 12:00. While the models are in agreement to the 10 per cent level, the LFSM exhibits an unexpected dip at $\sim 45 \mathrm{MHz}$. After subtraction of a fifth-order polynomial in $\log v$ (bottom panel), a discontinuity can be seen in the GSM2016 residual data. The residuals for both the GSM2016 and LFSM are of order $\sim 100 \mathrm{~K}$, notably larger than the GSM. The behaviour can be traced to the inclusion of data from the Alvarez et al. (1997) 45-MHz survey, suggesting a systematic offset in the underlying data from which the sky model is generated. Due to the unexpected discrepancies in the GSM2016 and LFSM data, we use the GSM2008 as our reference model. 

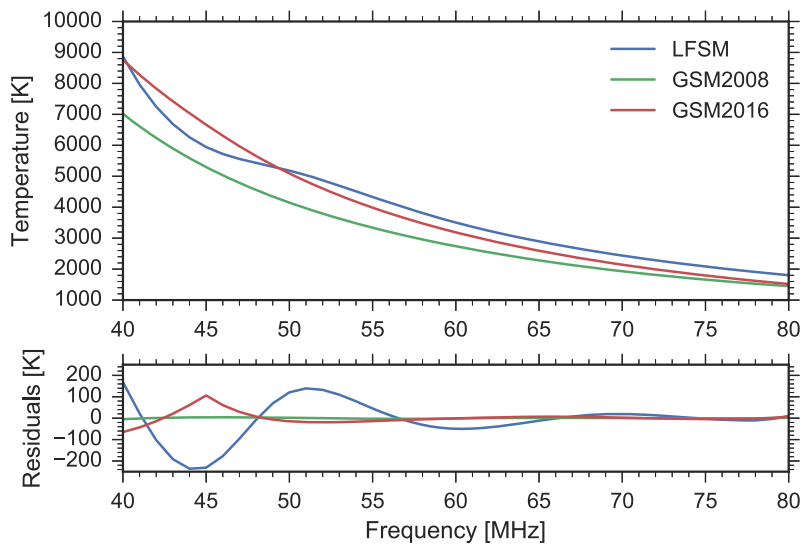

Figure 25. Top: simulated sky brightness for models of diffuse emission at LST 12:00 (top). A model of the LWA1 antenna is convolved with the sky models for an observer at OVRO-LWA. Bottom: residuals after subtraction of a fifth-order log-polynomial fit.
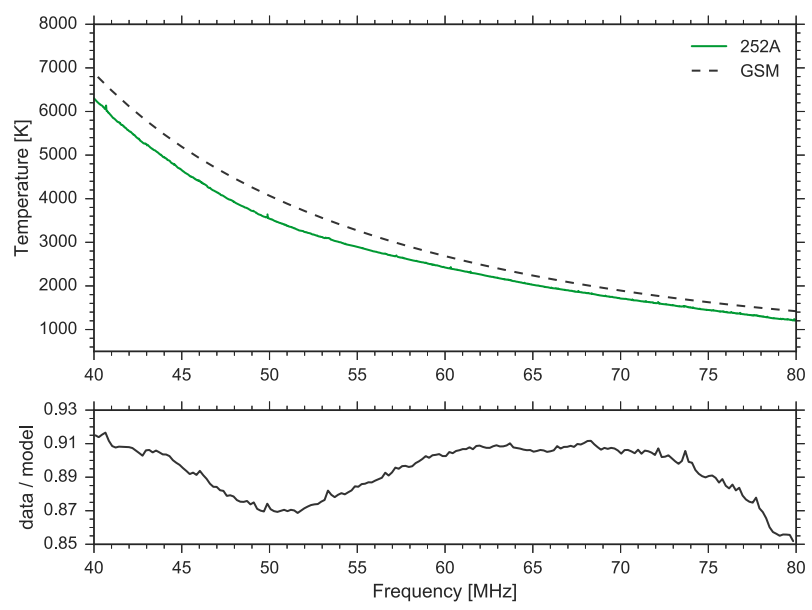

Figure 26. Comparison of simulated data (GSM2008 sky model with LWA antenna model) to calibrated data from antenna 252A, LST 11:00.

A comparison between calibrated spectrum and that expected from the GSM2008 is shown in Fig. 26. The ratio between data and model lies between 0.85 and 0.92 across the $40-80 \mathrm{MHz}$ band.

\subsection{Spectral index}

To compute the spectral index $\alpha$, we perform a least-squares minimization on

$\chi^{2}=\sum_{i}^{N} \frac{\left[T_{\mathrm{i}}^{\text {meas }}-T_{70}\left(\frac{\nu_{\mathrm{i}}}{70 \mathrm{MHz}}\right)^{\alpha}\right]^{2}}{\sigma_{\mathrm{i}}^{2}}$

where $T_{\mathrm{i}}^{\text {meas }}$ are our measured sky temperature data per frequency channel $v_{i}$, and $\sigma_{i}^{2}$ are per-channel estimates of the thermal noise. We used the LMFIT PYTHON package perform the minimization of $\chi^{2}$ over fit parameters $T_{70}$ and $\alpha$. We find the spectral index varies between -2.28 to -2.38 over LST (Fig. 27). These values are consistent with other Northern hemisphere experiments (Table 3). The effect of beam chromaticity (Mozdzen et al. 2017) is not considered here, and is left for future work.

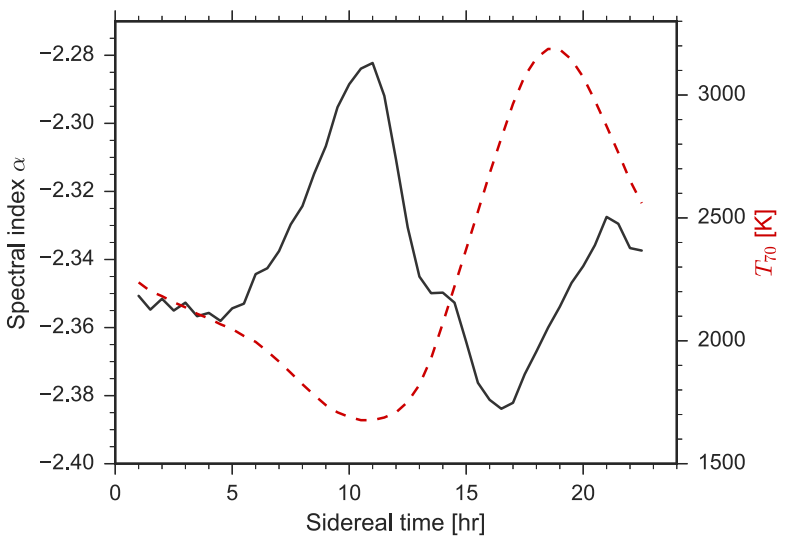

Figure 27. Best-fitting parameters for spectral index $\alpha$ and $T_{70}$ as a function of LST.

\subsection{Comparisons across antennas}

Figure 28 shows calibrated sky temperatures for antennas 252A, $254 \mathrm{~A}$ and $255 \mathrm{~A}$ as a function of frequency and sidereal time over a 24-hour period. The fractional difference between spectra integrated for $20 \mathrm{~min}$ around LST 12:00 are shown in Fig. 29; measurements are consistent to \pm 5 per cent between 40 and $83 \mathrm{MHz}$. Above $\sim 83 \mathrm{MHz}$, the attenuation due to bandpass filters gives rise to nonlinear ADC gain effects, which act to artificially attenuate the sky temperature (Backer 2007). As shown in Fig. 16, the receiver temperature also increases out of band. Improving the response above $83 \mathrm{MHz}$ is an ongoing effort toward verification of the purported (Bowman et al. 2018) absorption feature.

\subsection{Residuals across antennas}

As detailed in Bernardi et al. (2015) and Mozdzen et al. (2016), beam chromaticity must be accounted for to mitigate frequencydependent structure introduced to the global signal. The frequencydependent response of the antenna must therefore be either simulated using EM software packages such as HFSS and FEKO, or measured directly; LEDA employs the latter approach. In situ measurement of the gain pattern of LEDA antennas via cross-correlation with the OVRO-LWA core antennas is beyond the scope of this paper and will be detailed in a future publication.

Nevertheless, it is illustrative to subtract a log-polynomial sky model from the calibrated data to produce residuals. Fig. 30 shows the residuals after subtraction of log-polynomial fits for LEDA data between 50 and $80 \mathrm{MHz}$, averaged over a one-hour observation period centred an LST of 11:00, 2016-01-26. The calibration and reduction procedure was as follows. Data were calibrated following the absolute calibration approach of Section 5, after which RFI events were flagged (Section 7.2). After flagging, data were averaged in time ( $1 \mathrm{~h}$ total) and frequency $(1.008 \mathrm{MHz}$ bins) to form mean observed spectra, $T^{\text {meas }}(v)$ for each.

From top to bottom panel, Fig. 30 shows the residuals of calibrated data after subtraction of 1, 3, 5, and 7-term polynomial fits. We attribute differences between antenna stands primarily to beam chromaticity due to differences in surrounding terrain and differences in as-built antenna geometries. Antenna 252A exhibits the best performance (between -5 to $5 \mathrm{~K}$ after 7-term fit), with antennas $255 \mathrm{~A}$ and $255 \mathrm{~B}$ exhibiting notably higher residual values. 
Table 3. Experimental measurements of the spectral index of radio emission $\alpha$ below $200 \mathrm{MHz}$.

\begin{tabular}{lccc}
\hline Reference & $\begin{array}{c}\text { Declination } \\
(\text { deg })\end{array}$ & $\begin{array}{c}\text { Frequency } \\
\text { (MHz) }\end{array}$ & $\alpha$ \\
\hline Costain (1960) & +52.16 & $38-178$ & $-2.37 \pm 0.04$ \\
Purton (1966) & & $13-100$ & $-2.38 \pm 0.05$ \\
Andrew (1966) & +52.16 & $10-38$ & $-2.43 \pm 0.03$ \\
Rogers \& Bowman (2008) & -26.5 & $100-200$ & $-2.5 \pm 0.1$ \\
Patra et al. (2017) & +13.6 & $110-175$ & -2.30 to -2.45 \\
Mozdzen et al. (2017) & -26.7 & $90-190$ & -2.5 to -2.6 \\
This work & +37.24 & $40-80$ & -2.28 to -2.38 \\
\hline
\end{tabular}
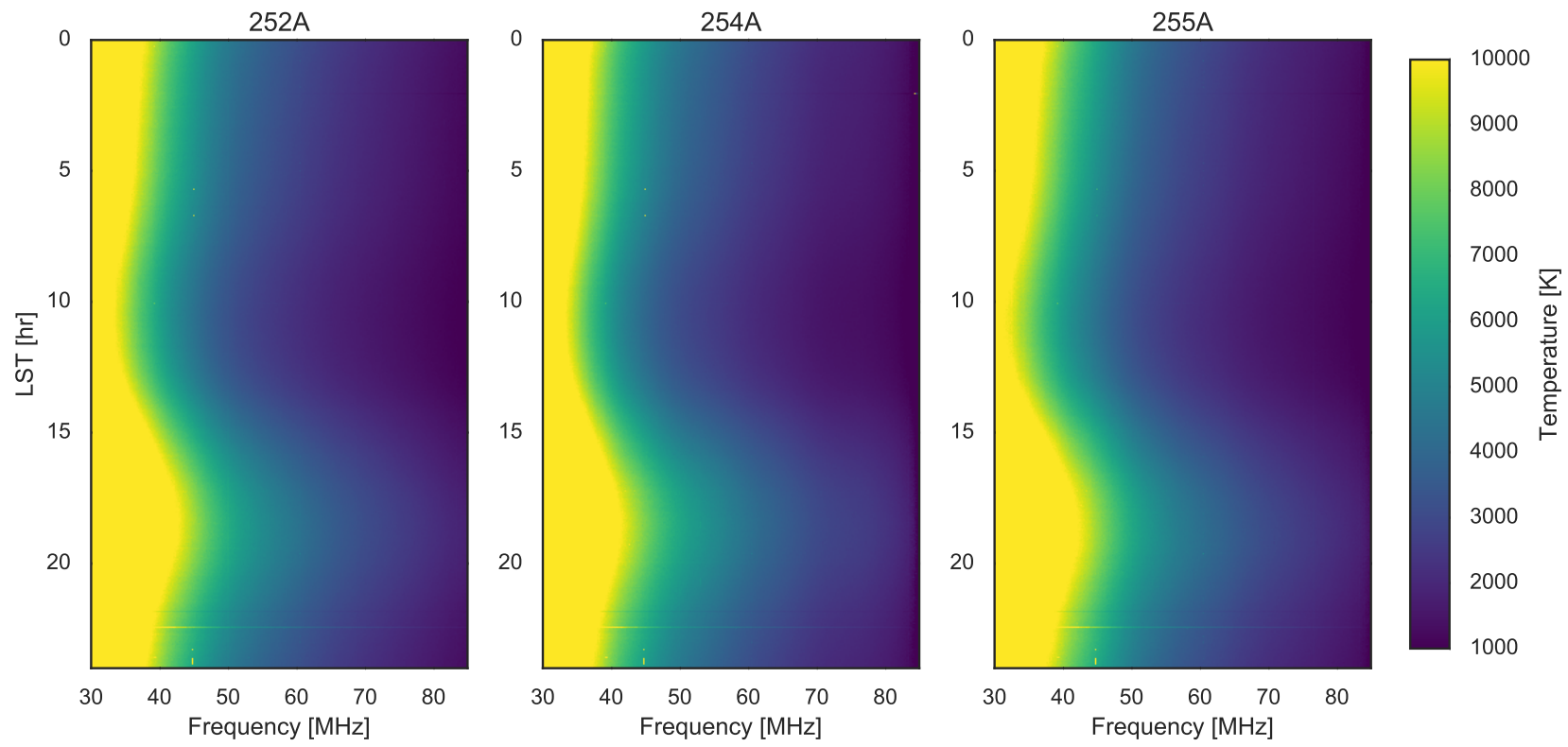

Figure 28. Calibrated sky temperature OVRO-LWA on 2016 January 27, as measured with three independent radiometer systems. Each dynamic spectra shows the sky temperature as a function of frequency over $24 \mathrm{~h}$ of local sidereal time.
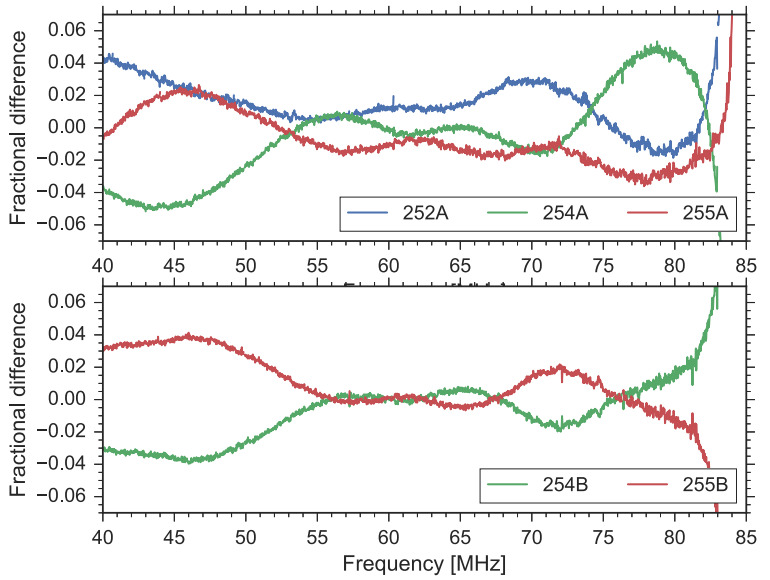

Figure 29. Fractional difference between calibrated spectra as shown in Fig. 28.

\section{DISCUSSION}

In this paper, we have presented the design and preliminary characterization results for the LEDA radiometer systems. The path toward detection of the $21-\mathrm{cm} \mathrm{CD}$ trough will require iterative improve- ments of the analogue systems and analysis methods, as knowledge of the instrumental systematics improve.

By comparison to the GSM2008, we find our antenna temperature is within 10-15 per cent of that predicted for an empirical model of the LWA antenna. Unaccounted for losses in the antenna, or inaccuracy of the manufacturer-supplied specifications of the HP346C noise source could account for this; if a multiplicative scale factor of 1.12 is applied, measured data agree with the model to within \pm 3 per cent.

We measure the spectral index of the sky to vary between -2.28 (LST 11:00) to -2.38 (LST 17:00, when the galaxy is high). While in agreement with other observations, we note that beam chromaticity has not been accounted for, which would improve the measurement. Pickup from the ground due to an imperfect ground screen, and from the Sierra Nevada mountain range on the horizon, potentially flatten the true spectral index of the radio sky. Improved measurement of the spectral index is the subject of future work.

\subsection{In-situ beam measurements}

An important outstanding step is empirical measurement of the antenna gain pattern for each outrigger antenna. As discussed in Bernardi et al. (2015) and Mozdzen et al. (2016), gain-patterninduced chromaticity limits foreground subtraction: this motivated 
Pol A
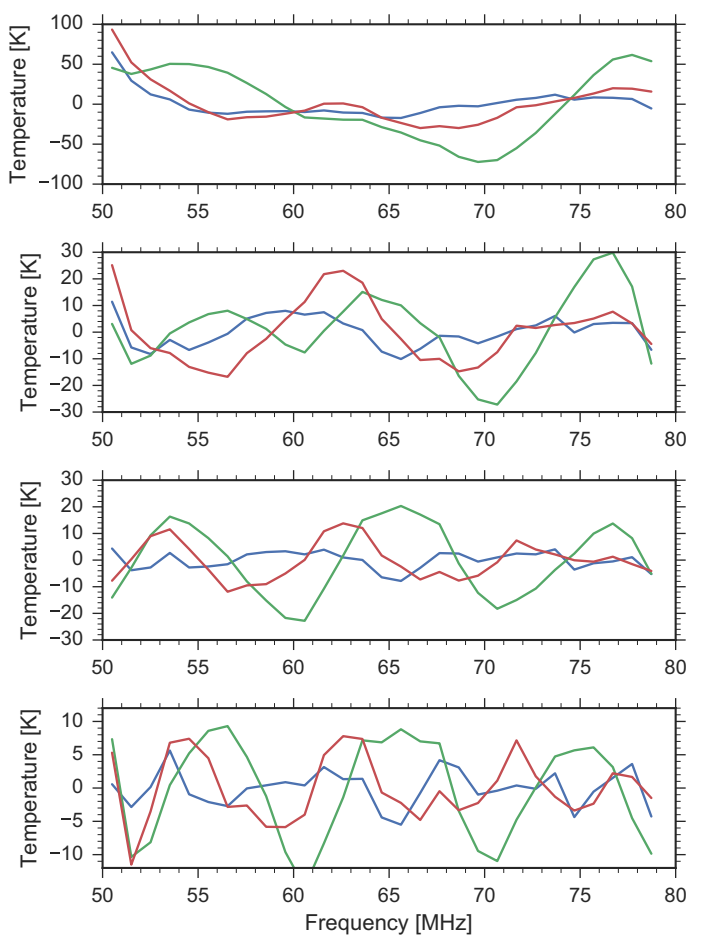

Pol B
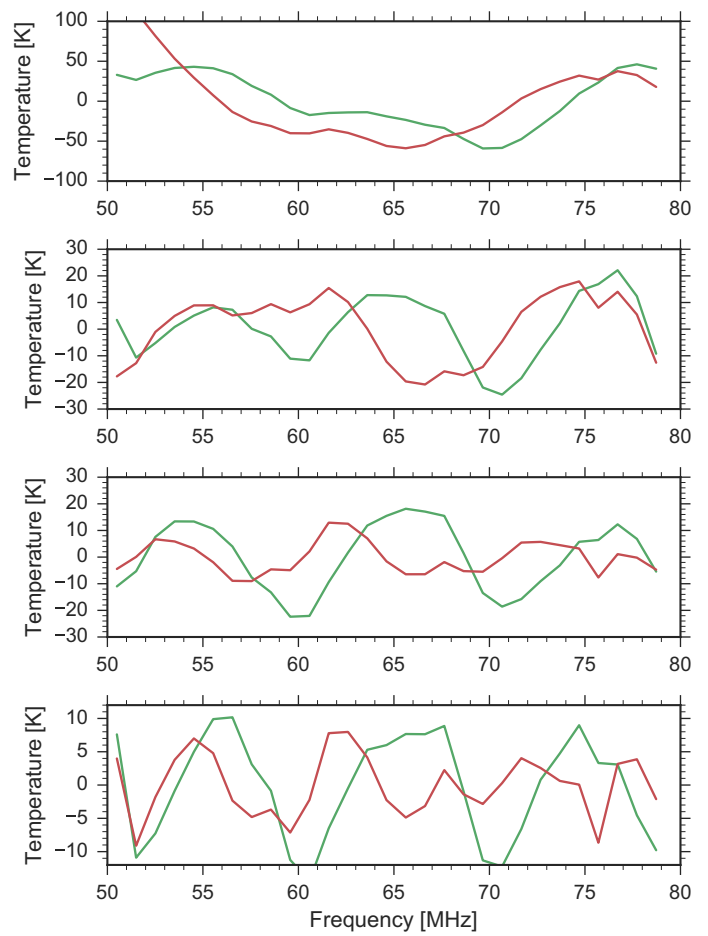

Figure 30. Top panel: residuals for calibrated data after subtraction of 1, 3, 5, and 7-term log-polynomial fits, for antennas 252 (blue), 254 (green), and 255 (red); polarization A is shown on the left and polarization B on the right.

a complete redesign of the EDGES antenna to improve chromatic performance. While an empirical beam model for (closely packed) LWA antennas has been derived by Dowell et al. (2017), calibration requirements motivate a per-antenna model. Measurements between three antennas (Section 7.5) show agreement to within \pm 5 per cent between 40 and $83 \mathrm{MHz}$; variation in antenna gain pattern may account for much of this. Indeed, these data highlight the advantage that the redundancy offered by multiple measurements of the sky with different radiometer systems provides.

\subsection{Future improvements}

We identify several areas in which our instrument characterization can be improved. First, periodic measurement of scattering parameters measured in the field would allow longitudinal monitoring of the receiver and antenna's reflection coefficients. Additionally, the emitted noise waves could also be measured in the field. Measurement of the noise waves using a commercial impedance tuner in lieu of an open cable would offer an alternative characterization of the LNA noise waves.

Here, we applied the calibration formalism of Rogers \& Bowman (2012). Other approaches, such as the matrix-based calibration approach of King (2010) and King et al. (2015) offer an alternative approach based on more modern formalisms of noise characteristics. The approach of Monsalve et al. (2017), in which extra calibration parameters are included to better fit the data, also offers an alternative avenue toward improved instrument modelling.

Our absolute temperature calibration relies upon an HP346C noise source with manufacturer-supplied characterization. Crosscalibration with other calibration standards, and/or experimental verification of the manufacturer-supplied parameters, may provide improved accuracy of the absolute temperature scaling.

\subsection{Validation of EDGES absorption feature}

Validation of the absorption feature reported by Bowman et al. (2018) is pressing. As reported here, the LEDA systems exhibit the required radiometric stability, but other systematics, namely the direction-dependent gain of the antennas, confound measurement. Further characterization work is ongoing.

Upgrades to the LEDA systems are made on a rolling basis. Since the 2016 January campaign - as detailed here - several upgrades have been made to the LEDA systems. These improvements will be discussed further in a future paper. Briefly, radiometric receivers have been installed on all five outrigger antennas, modifications to further improve the stability of the noise diode have been made, and a logging system for measurement of the ambient temperature at the antenna has been added. Of importance to validation of the Bowman et al. (2018) signal, bandstop filters with sharper roll-off have been sourced to allow access to frequencies of up to $87.5 \mathrm{MHz}$, while still strongly attenuating the $88-108 \mathrm{MHz}$ FM band.

An observation campaign with the upgraded LEDA system was undertaken over 2016 November-2017 March; analysis of these data, along with data from 2018, is ongoing.

\section{CONCLUSIONS}

Measurement of the 21-cm emission from the early Universe via radiometric methods requires exquisite calibration and comprehensive knowledge of the radiometer systems. The purported detection of a 21-cm absorption feature during Cosmic Dawn by Bowman et al. (2018) suggests that the radiometric approach does indeed offer a window into Cosmic Dawn. Validation of the Bowman et al. (2018) signal is pressing, particularly given the concerns raised by 
Hills et al. (2018), and is an exciting opportunity for radiometric Cosmic Dawn experiments such as LEDA, SARAS 2, and PRIZM.

In this paper, we have presented the design and characteristics of the LEDA radiometer systems. Comparison of the system performance with predictions based on the GSM2008 sky model and LWA antenna gain pattern are in agreement to the 15 per cent level over $40-83 \mathrm{MHz}$. Between antennas, data agree to \pm 5 percent. Above $83 \mathrm{MHz}$, the rolloff of the filter for FM-band rejection (88$108 \mathrm{MHz}$ ) becomes significant.

Upgrades to increase the LEDA observation window cut-off from 83 to $87.5 \mathrm{MHz}$ are underway. Further characterization work is also ongoing, in order to place limits on the 21-cm emission during Cosmic Dawn. In particular, individual characterization of the antenna's direction-dependent gain may be needed to account for the frequency dependence of the beam. Work on this characterization is underway, using interferometric measurements with the combined LEDA radiometer antennas and OVRO-LWA core antennas.

\section{ACKNOWLEDGEMENTS}

This work has benefited from open-source technology shared by the CASPER. We thank the Xilinx University Program for donations; NVIDIA for proprietary tools, discounts, and donations; Digicom for collaboration on manufacture and testing of samplers and $\mathrm{ROACH} 2$ processors; and Y. Belopolsky (Bel-Stewart R\&D) for collaboration in development of CAT-7A ARJ45 pass-through hardware and cable assemblies. We thank R. Blundell, and P. Riddle of the Smithsonian Astrophysical Observatory Submillimeter Receiver Lab for collaboration on development and fabrication of receivers and other LEDA signal path and control elements. The great dedication, innovation, and exemplary skill of the Caltech Owens Valley Radio Observatory staff, demonstrated in constructing the LWA array, having created a purpose-built facility in no time deserves special mention. LEDA research has been supported in part by NSF grants AST/1106059, PHY/0835713, and OIA/1125087. The OVRO-LWA project was enabled by the kind donation of Deborah Castleman and Harold Rosen. GB acknowledges support from the Royal Society and the Newton Fund under grant NA150184. This work is based on the research supported in part by the National Research Foundation of South Africa under grant 103424. GH acknowledges the support of NSF CAREER award AST/1654815.

\section{REFERENCES}

Ali Z. S. et al., 2015, ApJ, 809, 61

Alvarez H., Aparici J., May J., Olmos F., 1997, A\&AS, 124, 315

Andrew B. H., 1966, MNRAS, 132, 79

Atek H., et al. 2015, ApJ, 814, 69

Backer D., 2007, Technical Report, Quantization with Four Bits, CASPER Memo (16), March 2007

Barkana R., 2016, Phys. Rep., 645, 1

Barkana R., 2018, Nature, 555, 71

Beardsley A. P. et al., 2016, ApJ, 833, 102

Becerra F., Greif T. H., Springel V., Hernquist L. E., 2015, MNRAS, 446, 2380

Bernardi G. et al., 2016, MNRAS, 461, 2847

Bernardi G., McQuinn M., Greenhill L. J., 2015, ApJ, 799, 90

Bouwens R. J. et al., 2015, ApJ, 803, 34

Bouwens R. J. et al., 2016, ApJ, 833, 72

Bowman J. D., Rogers A. E. E., Hewitt J. N., 2008, ApJ, 676, 1

Bowman J. D., Rogers A. E. E., Monsalve R. A., Mozdzen T. J., Mahesh

N., 2018, Nature, 555, 67

Bromm V., Yoshida N., 2011, ARA\&A, 49, 373
Burns J. O. et al., 2017, ApJ, 844, 33

Clark M. A., Plante P. L., Greenhill L. J., 2013, Int. J. High Perform. Comput. Appl., 27, 178

Cohen A., Fialkov A., Barkana R., Lotem M., 2017, MNRAS, 472, 1915

Costain C. H., 1960, MNRAS, 120, 248

Datta A., Bradley R., Nhan B. D., Burns J. O., 2014, Radio Science Meeting (USNC-URSI NRSM), 2014 United States National Committee of URSI National, p. 1

de Oliveira-Costa A., Tegmark M., Gaensler B. M., Jonas J., Landecker T. L., Reich P., 2008, MNRAS, 388, 247

DeBoer D. R. et al., 2017, PASP, 129, 045001

Dowell J., Taylor G., 2018, ApJ, 858, L9,

Dowell J., Taylor G. B., Schinzel F. K., Kassim N. E., 2017, MNRAS, 469, 4537

Ellingson S. et al., 2013, IEEE Trans. Antennas Propag., 61, 2540

Ewall-Wice A. et al., 2016, MNRAS, 460, 4320

Fialkov A., Loeb A., 2016, ApJ, 821, 59

Fialkov A., Barkana R., Visbal E., 2014, Nature, 506, 197

Field G. B., 1958, Proc. IRE, 46, 240

Greenhill L., 2018, Nature, 555, 38

Greenhill L. J., Bernardi G., 2012, preprint (arXiv:1201.1700)

Greif T. H., 2015, Comput. Astrophys. Cosmol., 2, 3

Haiman Z., 2016, in Astrophysics and Space Science Library, Vol. 423, Understanding the Epoch of Cosmic Reionization: Challenges and Progress, Springer International Publishing, Switzerland, p. 1

Harker G. J. A., Pritchard J. R., Burns J. O., Bowman J. D., 2012, MNRAS, 419,1070

Hickish J. et al., 2016, J. Astron. Instrum., 5, 1641001

Hills R., Kulkarni G., Meerburg P. D., Puchwein E., 2018, preprint (arXiv: 1805.01421)

Hirano S., Bromm V., 2017, MNRAS, 470, 898

King O. G. et al., 2015, MNRAS, 446, 2

King O. G., 2010, in Twenty-First International Symposium on Space Terahertz Technology, Proceedings of a meeting held in Oxford, UK, 2010 March 23-25, p. 379

Kocz J. et al., 2014, J. Astron. Instrum., 03, 1450002

Kocz J. et al., 2015, J. Astron. Instrum., 4, 1550003

Koopmans L. et al., 2015, in Bourke T. L. et. al. eds, Advancing Astrophysics with the Square Kilometre Array (AASKA14), 2014 June 9-13. Giardini Naxos, Italy, p. 1

Laporte N. et al., 2017, ApJ, 837, L21

Mesinger A., Greig B., Sobacchi E., 2016, MNRAS, 459, 2342

Meys R. P., 1978, IEEE Trans. Microw. Theor. Tech., 26, 34

Monsalve R. A., Rogers A. E. E., Bowman J. D., Mozdzen T. J., 2017, ApJ, 835,49

Mortlock D. J. et al., 2011, Nature, 474, 616

Mozdzen T. J., Bowman J. D., Monsalve R. A., Rogers A. E. E., 2016, MNRAS, 455,3890

Mozdzen T. J., Bowman J. D., Monsalve R. A., Rogers A. E. E., 2017, MNRAS, 464, 4995

Offringa A. R., de Bruyn A. G., Biehl M., Zaroubi S., Bernardi G., Pandey V. N., 2010, MNRAS, 405, 155

Paciga G. et al., 2013, MNRAS, 433, 639

Patil A. H. et al., 2017, ApJ, 838, 65

Patra N., Subrahmanyan R., Raghunathan A., Udaya Shankar N., 2013, Exp. Astron., 36, 319

Patra N., Bray J. D., Roberts P., Ekers R. D., 2017, Exp. Astron., 43, 119

Planck Collaboration et al., 2016, A\&A, 596, A108

Pober J. C. et al., 2015, ApJ, 809, 62

Pozar D. M., 2005, Microwave Engineering, 3rd edn. John Wiley and Sons Inc., New York

Pritchard J. R., Loeb A., 2010, Phys. Rev. D, 82, 023006

Purton C. R., 1966, MNRAS, 133, 463

Robertson B. E., Ellis R. S., Furlanetto S. R., Dunlop J. S., 2015, ApJ, 802, L19

Rogers A. E. E., Bowman J. D., 2008, AJ, 136, 641

Rogers A. E. E., Bowman J. D., 2012, Radio Sci., 47, 0K06 
Safranek-Shrader C., Montgomery M. H., Milosavljević M., Bromm V., 2016, MNRAS, 455, 3288

Schinzel F. K. et al. 2018, A Prototype Lower-VHF Radiometry System at the LWA, Long Wavelength Array Memo 208

Shaver P. A., Windhorst R. A., Madau P., de Bruyn A. G., 1999, A\&A, 345, 380

Singh S. et al., 2017, ApJ, 845, L12

Singh S., Subrahmanyan R., Shankar N. U., Rao M. S., Girish B. S., Raghunathan A., Somashekar R., Srivani K. S., 2018, Exp. Astron., 45, 269

Smidt J., Whalen D. J., Johnson J. L., Li H., 2017, preprint (arXiv:1703.0 0449)

Smith A., Bromm V., Loeb A., 2017, Astron. Geophys., 58, 3.22

Sokolowski M. et al., 2015, PASA, 32, e004

Taylor G. B. et al., 2012, J. Astron. Instrum., 1, 1250004
Voytek T. C., Natarajan A., Jáuregui García J. M., Peterson J. B., López-Cruz O., 2014, ApJ, 782, L9

Wallin A. E. E., Price D. C., Carson C. G., Meynadier F., 2018, Astrophysics Source Code Library, record ascl:1804.021

Wise J. H., Demchenko V. G., Halicek M. T., Norman M. L., Turk M. J., Abel T., Smith B. D., 2014, MNRAS, 442, 2560

Wouthuysen S. A., 1952, AJ, 57, 31

Wu X.-B. et al., 2015, Nature, 518, 512

Zheng H. et al., 2017, MNRAS, 464, 3486

This paper has been typeset from a $\mathrm{T}_{\mathrm{E}} \mathrm{X} / \mathrm{LT} \mathrm{E} \mathrm{X}$ file prepared by the author. 